\title{
The Fluorescent Protein Color Palette
}

\author{
Scott G. Olenych, Nathan S. Claxton, Gregory K. Ottenberg, and Michael W. Davidson* \\ National High Magnetic Field Laboratory, 1800 East Paul Dirac Drive, The Florida State \\ University, Tallahassee, Florida 32310-3706
}

\begin{abstract}
Advances in fluorescent protein development over the past 10 years have led to fine-tuning of the Aequorea victoria jellyfish color palette in the emission color range from blue to yellow, while a significant amount of progress has been achieved with reef coral species in the generation of monomeric fluorescent proteins emitting in the orange to far-red spectral regions. It is not inconceivable that near-infrared fluorescent proteins loom on the horizon. Expansion of the fluorescent protein family to include optical highlighters and FRET biosensors further arms this ubiquitous class of fluorophores with biological probes capable of photoactivation, photoconversion, and detection of molecular interactions beyond the resolution limits of optical microscopy. The success of these endeavors certainly suggests that almost any biological parameter can be investigated using the appropriate fluorescent protein-based application.
\end{abstract}

Keywords: GFP, fluorescent proteins, optical highlighters, biosensors, photoconversion, photoactivation, chromophore, fluorophore, FRET, FRAP, microscopy, confocal

\section{INTRODUCTION}

Although the first report of fluorescence emission in the bioluminescent hydrozoan jellyfish species Aequorea victoria was recorded in the mid-twentieth century (Davenport and Nichol, 1955) and a protein extract was independently demonstrated by two investigators to be responsible for this "green" fluorescence in the 1960s and 1970s (Shimomura, et al., 1962; Morin and Hastings, 1971), it took another 20 years and a number of significant advances in the technology of molecular and cellular biology to witness the elucidation of the primary amino acid structure (Prasher, et al., 1992). Following shortly thereafter was the astonishing demonstration that the jellyfish green fluorescent protein (GFP) could be readily employed as a useful marker for gene expression (Chalfie, et al., 1994) in cells evolutionarily far removed from the jellyfish. During the next several years, a number of "enhanced" genetic variants having fluorescence emission spectral profiles in the blue (Yang, et al., 1998), cyan (Heim and Tsien, 1996), green (Heim, et al., 1995), and yellow (Wachter, et al., 1998) regions of the visible spectrum were developed by engineering specific mutations of the original GFP nucleotide sequence. One of the most significant advances following the initial cloning and early mutagenesis efforts on the Aequorea victoria green fluorescent protein was the discovery of similar proteins in non-bioluminescent reef corals and sea anemones (Matz, et al., 1999; Shagin, et al., 2004) that not only provided a large spectrum of new emission colors, but also demonstrated that these protein motifs occur in a wide range of organisms.

Over the past decade, fluorescent proteins have launched a new and unprecedented era in cell biology by enabling investigators to apply routine molecular cloning methods, fusing these optical probes to a wide variety of protein and enzyme targets, in order to monitor cellular processes in living systems using fluorescence microscopy and related methodology (for recent reviews, see: Chudakov, et al., 2005; Miyawaki, et al., 2005; Shaner, et al., 2005). The spectrum of applications for fluorescent proteins ranges from reporters of transcriptional regulation and targeted markers for organelles and other subcellular structures to fusion proteins designed to monitor motility and dynamics. These fascinating probes have also opened the door to creating biosensors for numerous intracellular phenomena, including $\mathrm{pH}$ and ion concentration fluctuations, protein kinase activity, apoptosis, voltage, and cyclic nucleotide signaling (Meyer and Teruel, 2003; Miyawaki, 2003; Zaccolo, 2004; Zhang, et al., 2002). By applying selected 
promoters and targeting signals, fluorescent protein biosensors can be introduced into an intact organism and directed to a host of specific tissues, cell types, as well as subcellular compartments to enable an unprecedented focus on monitoring a variety of physiological processes.

When coupled to recent technical advances in widefield fluorescence and confocal microscopy, including ultra-fast low light level digital CCD cameras, spinning disk and swept-field instruments, as well as multitracking laser control systems with acousto-optic tunable filter (AOTF) control, the green fluorescent protein and its color-shifted genetic derivatives have demonstrated invaluable service in many thousands of live-cell imaging experiments. Compared to many traditional synthetic fluorophores, which are often toxic or photoreactive, the use of fluorescent proteins is minimally invasive for living cells, enabling visualization and recording of time-lapse image sequences for extended periods of time (Day, 2005; Lippincott-Schwartz and Patterson, 2003). Furthermore, continued advances in genetically finetuning the properties of fluorescent protein variants have led to increased brightness levels, improved photostability, and significantly better expression in mammalian cells (Shaner, et al., 2005; Verkhusha and Lukyanov, 2004). These functional enhancements have stimulated a wide variety of investigations into protein dynamics and function using fluorescent protein chimeras imaged at low light intensities for many hours to extract valuable information about changes in the steady-state distribution.

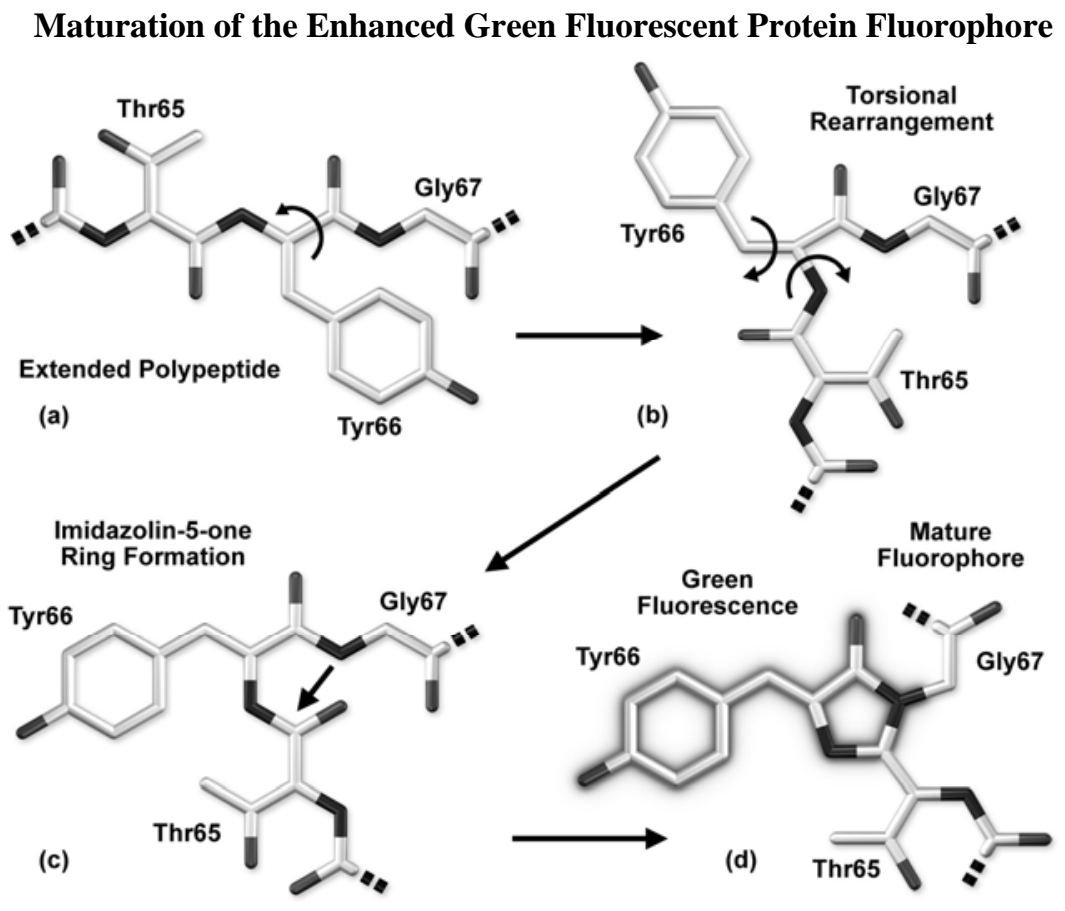

Figure 1. Schematic diagram of the chromophore formation in maturing enhanced green fluorescent protein (EGFP). (a) The pre-maturation EGFP fluorophore tripeptide amino acid sequence (Thr65-Tyr66-Gly67) stretched into a linear configuration so that the threonine residue is positioned in the upper left-hand corner of the diagram. The first step in maturation is a series of torsional adjustments (b) and (c) that relocate the carboxyl carbon of Thr65 so that it is in close proximity to the amino nitrogen of Gly67. Nucleophilic attack by this carbon atom on the amide nitrogen of glycine, followed by dehydration, results in formation of an imidazolin-5-one heterocyclic ring system. (d) Fluorescence occurs when oxidation of the tyrosine $\alpha-\beta$ carbon bond by molecular oxygen extends electron conjugation of the imidazoline ring system to include the tyrosine phenyl ring and its para-oxygen substituent.

Among the most noteworthy attributes of the original green fluorescent protein derived from the Aequorea victoria jellyfish, as well as the more recently developed palette of color-shifted genetic variants, is that a stable and highly-defined cylindrical polypeptide structure is essential for the development and maintenance of fluorescence in this very remarkable family of proteins (Rizzo and Piston, 2005a). The principle fluorophore (often termed a chromophore) in GFP is a tripeptide consisting of the residues serine, 
tyrosine, and glycine at positions $65-67$ in the sequence. Although this simple amino acid motif is commonly found throughout nature, it does not generally result in fluorescence. The chromophore forms spontaneously after translation without the requirement for cofactors or external enzyme components (other than molecular oxygen), through a self-catalyzed intramolecular rearrangement of the tripeptide sequence to produce the fluorescent species (as illustrated in Figure 1 for the enhanced GFP variant). The first step in maturation is thought to be a series of torsional peptide and side-chain bond adjustments that relocate the carboxyl carbon of the serine amino acid at position 65 (Ser65) in close proximity to the amino nitrogen atom of Gly67. Nucleophilic attack by this carbon atom on the amide nitrogen of glycine, followed by dehydration, results in the formation of an imidazolin-5-one heterocyclic ring system (Cody, et al., 1993). Fluorescence occurs when oxidation of the tyrosine (Tyr66) $\alpha-\beta$ carbon bond by molecular oxygen extends electron conjugation of the imidazoline ring system to include the tyrosine phenyl ring and its para-oxygen substituent (Heim, et al., 1994). The result is a highly conjugated $\pi$-electron resonance system that largely accounts for the spectroscopic properties of the protein. Extensive mutagenesis studies suggest that the glycine residue at position 67 is critical in formation of the chromophore (Cubitt, et al., 1995; Delagrave, et al., 1995), and indeed, no fluorescent proteins have yet been discovered that lack this essential element.

Several fundamental physical and chemical aspects of the fluorescent protein chromophore have profound implications for the application of these biomolecules as intracellular probes. A striking aspect of the fluorescent protein chromophores is that although their presence to date has only been detected in sea creatures, they are able to form functional units in a wide variety of organisms ranging from bacteria and plants to mammals. The native Aequorea victoria GFP is composed of a single-domain protein with 238 amino acids and a molecular weight of approximately $27 \mathrm{kDa}$. Even though the photophysics of GFP are rather complex (Chattoraj, et al., 1996; Jung, et al., 2005), the molecular structure of the protein is quite robust and can accommodate a high degree of modification (executed through the amino acid sequence) without destroying the ability to emit fluorescence. A wide variety of amino acid substitutions to the original configuration have been successful in fine-tuning the fluorescence of native GFP to provide a broad range of derivative fluorophores that emit colors traversing from the blue to the yellow regions of the visible spectrum (Shaner, et al., 2005; Tsien, 1998). Site-directed and random mutagenesis investigations (Heim and Tsien, 1995; Tsien, 1998; Zacharias and Tsien, 2006) have revealed that fluorescence is very dependent on the three-dimensional structure of amino acid residues surrounding the chromophore. Denaturation of the protein, as might be expected, destroys fluorescence (Alkaabi, et al., 2005; Ward, 2006), and mutations in residues immediately adjacent to the chromophore can significantly alter the fluorescent properties. Surprisingly, amino acid substitutions in regions of the polypeptide far removed from the chromophore can also affect the spectral characteristics of the protein (Zacharias and Tsien, 2006).

The remarkably well-conserved cylindrical geometry of all the fluorescent proteins discovered thus far appears to be ideally suited to the primary function of protecting the chromophore. Formed in the shape of a cylinder (Ormö, et al., 1996) having dimensions of approximately 25 x $40 \AA$, the polypeptide backbone is wound into 11 strands of an extensively hydrogen bonded beta-sheet that surround a central alpha-helix containing the chromophore with short helical segments protruding from the ends of the cylinder. Dubbed a "beta-can" because of the geometrical symmetry (Yang, et al., 1996), the tight packing of amino acid residues bestows a high level of stability to the protein, which often results in relatively large fluorescent quantum yields for GFP and its derivatives (up to 80\%). A lack of clefts and gaps for access of small ligands (such as ions and oxygen), combined with the fact that the chromophore is located near the exact center of the protein, partially explains the extraordinary photostability and high quantum yields that are observed. In addition, both the N- and C- termini are "conveniently" exposed on the surface of the beta-can to readily participate in protein fusions that do not affect the structural integrity. This consolidated protein organizational motif also enhances resistance to changes in $\mathrm{pH}$, temperature, fixation with paraformaldehyde, and the disruptive action of many common denaturing agents, such as urea and guanidine hydrochloride. Mutations in the amino acid sequence that affect the fluorescence profile generally also produce negative effects on overall stability, usually resulting in a reduction of quantum yield, increased rates of photobleaching, and enhanced environmental sensitivity. 


\section{Fluorescent Protein Brightness and Maturation}

When designing experiments using fluorescent protein reporters, investigators should ensure that the highest possible signal levels are achieved, especially in cases where the targeted species is rapidly turned over or exhibits low overall population abundance. The apparent brightness of a fluorescent protein, a seemingly qualitative concept, is determined by a number of factors that include the efficiency and rate of maturation, the level of protein expression, the molar extinction coefficient values within the excitation wavelength range, and the quantum yield (Shaner, et al., 2005; Tsien, 1998; Ward, 2005). In addition, the fluorescence filter combination, spectral distribution of the illumination source, and the digital camera parameters utilized for imaging of fluorescent proteins are of paramount importance in determining whether adequate contrast and signal-to-noise ratios can be achieved.

The intrinsic "brightness" of a fluorescent protein is determined by the product of the molar extinction coefficient at the peak of the absorption band and the integrated emission quantum yield (Rizzo and Piston, 2005a; Shaner, et al., 2005). As an example, if two proteins have identical quantum yields but one has twice the extinction coefficient at the wavelength of excitation, then the protein with the largest extinction coefficient would have double the brightness of the protein with the smaller extinction coefficient. Likewise, in fluorescent proteins with similar molar extinction coefficients, the one with the highest quantum yield will be the brightest. Quantitative assessment of extinction coefficients and quantum yields is a tedious process that requires a highly purified and correctly folded protein with greater than $95 \%$ of the molecules having an active fluorescent chromophore (Ward, 2006). In addition, the total protein concentration must be accurately determined and the measurements of absorption and fluorescence emission performed in a reliable, calibrated spectrophotometer and fluorimeter. Quantum yield assessment requires the comparison of emission spectra between the unknown fluorescent protein and a reference standard having a similar wavelength profile. Investigators should be skeptical of purely qualitative fluorescent protein brightness evaluations (often made by commercial distributors) that lack quantitative information pertaining to the extinction coefficient and quantum yield. It is difficult, if not impossible, to accurately perform brightness comparisons between fluorescent proteins without knowledge of these critical parameters.

Fluorescent protein maturation efficiency is also determined by a number of variables, including the origin of the protein and the abundance of species-specific optimum codons in the organism chosen for expression. In general, fluorescent proteins that have been codon-optimized for expression in mammalian cells will be expressed with high efficiency and mature rapidly at $37^{\circ} \mathrm{C}$, although a small percentage of the product may not fold correctly, resulting in poor targeting and high background fluorescence. Many of the protein variants derived from the original Aequorea jellyfish green fluorescent protein have been optimized (with regards to the wild-type GFP) through mutagenesis for expression in mammalian systems (Nagai, et al., 2002; Shaner, et al., 2005; Tsien, 1998; Zapata-Hommer, et al., 2003) at high efficiency. Fluorescent proteins from reef corals and sea anemones generally express well at $37^{\circ} \mathrm{C}$, presumably because the native species from which the proteins are obtained have evolved in warmer habitats (Salih, et al., 2000). The presence of molecular oxygen is also a critical factor in fluorescent protein chromophore development during the maturation process. During the formation of chromophores in Aequorea protein variants, at least one oxygen molecule is required for dehydrogenation, while reef coral proteins that emit in the orange-red spectral regions usually require two molecules (Gross, et al., 2000; Tsien, 1998; Zimmer, 2002). In mammalian cell cultures, fluorescent protein expression is rarely hampered by a lack of oxygen, but anoxia could become a limiting factor in other systems.

Regardless of the intrinsic brightness displayed by a particular fluorescent protein, the ability to achieve high signal levels is primarily determined by the configuration of the imaging equipment. The laser system or arc-discharge lamp coupled to fluorescence filters used to excite the chromophore should strongly overlap the chromophore absorption profile and the emission filters must have the widest possible bandpass region coinciding with the emission spectrum. In addition, the camera system must be capable of recording images with high quantum efficiency in the fluorescence emission region of interest (Patterson, et al., 1997), and the optical system of the microscope should have high throughput in the wavelength regions necessary for producing excitation and gathering emission. Even with research-level instrumentation, it is often quite difficult to achieve the maximum potential fluorescent protein brightness levels in each spectral 
class unless the fluorescence filter sets are completely optimized for imaging the proteins. Many core imaging facilities have limited inventories of filter sets that are typically designed for traditional synthetic fluorophores rather than fluorescent proteins. For example, the standard "DAPI”, "FITC”, "TRITC”, and "Texas Red" fluorescence filter combinations, which are often marketed by default with widefield arcdischarge microscopes, are not suitable for many fluorescent proteins and are less than optimal for others.

\section{Fluorescence Filter Combinations for Fluorescent Proteins}
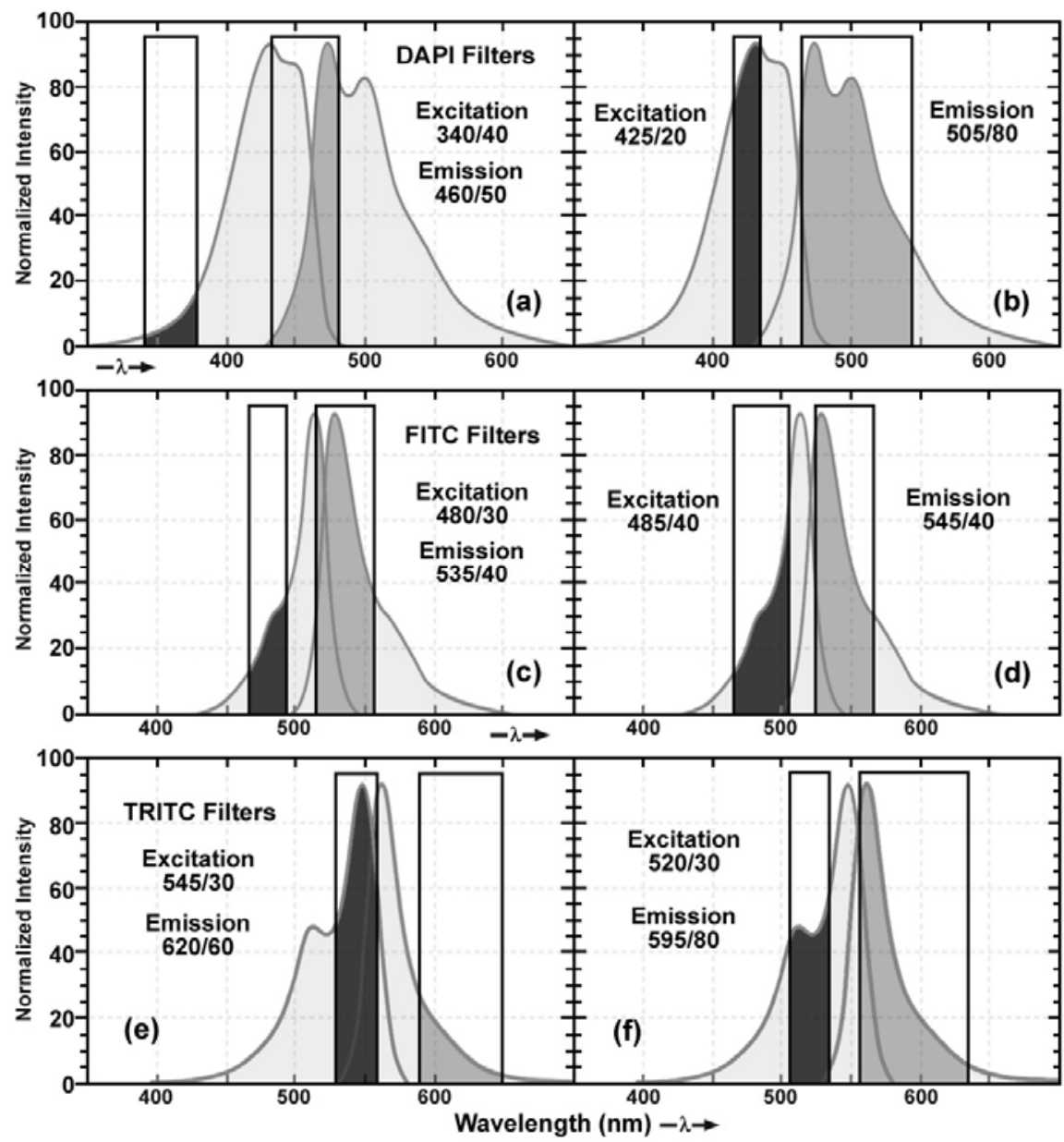

Figure 2. Spectral profiles of three common fluorescent proteins, Cerulean (a) and (b), Venus (c) and (d), and mKusabira Orange (e) and (f) superimposed over standard ultraviolet, blue, and green excitation filter combinations (a), (c), (e) and custom sets optimized to image the fluorescent proteins (b), (d), and (f). Filters are drawn as rectangles identifying the bandpass region and are described in terms of center wavelength and spectral bandpass.

The hazards of using incorrect filter sets for imaging fluorescent proteins are illustrated in Figure 2 for several of the more useful fluorescent proteins with "standard" widefield microscopy filter sets. The cyan fluorescent protein, Cerulean, is depicted in Figure 2(a) and 2(b) with the absorption and emission spectral profiles superimposed over a DAPI filter set (Figure 2(a)) as well as a set designed specifically for cyan fluorescent proteins (Figure 2(b)). Note the poor overlap of the DAPI filters with the Cerulean absorption and emission bands, which yields low signal when this combination is employed. Narrowing the excitation band to $20 \mathrm{~nm}$ and moving the center wavelength $85 \mathrm{~nm}$ to longer wavelengths significantly improves the excitation of Cerulean (Figure 2(b)). Likewise, the emission filter effectively captures a majority of the fluorescence emission with a wideband (80-nm) filter having a center wavelength of 505 $\mathrm{nm}$. In general, cyan fluorescent proteins are best imaged with custom filter sets specifically designed for 
their blue-violet excitation range. The standard FITC filter set is a much closer match for the absorption profile of the yellow fluorescent protein, Venus (Figure 2(c)), but a better combination increases the excitation filter bandwidth to $40 \mathrm{~nm}$ and moves the center wavelength to $485 \mathrm{~nm}$ (red-shifted by $5 \mathrm{~nm}$ ). The optimum emission filter bandwidth for Venus is similar to the FITC version with an increase of $10 \mathrm{~nm}$ for the center wavelength. Most of the green and yellow fluorescent proteins can be satisfactorily imaged with the FITC filter set, but quantitative imaging benefits from optimizing the central wavelengths and bandwidths for each protein. Orange fluorescent proteins have spectral profiles that fall between the FITC and TRITC filter wavelength regions for optimal excitation and gathering of emission. Imaging mKusabira Orange with a TRITC filter combination (Figure 2(e) and 2(f)) is a good example of the mismatch. The TRITC excitation filter covers an acceptable cross-section of the absorption spectrum, but is shifted slightly too far towards the emission wavelengths (Figure 2(e)). In addition, the emission filter for this set captures only a small portion of the fluorescent protein emission spectral "tail", leading to a loss of signal. A more effective filter set for mKusabira Orange is illustrated in Figure 2(f). Fortunately, a majority of the red fluorescent proteins (DsRed, mCherry, JRed, mPlum, etc.) can be imaged with good results using either the TRITC or Texas Red filter combinations.

\section{Phototoxicity and Photostability}

Aside from the toxicity that occurs due to excessive concentrations of synthetic fluorophores and the over-expression or aggregation of poorly localized fluorescent proteins, the health and longevity of optimally labeled mammalian cells in microscope imaging chambers can also suffer from a number of other deleterious factors. Foremost among these is the light-induced damage (phototoxicity) that occurs upon repeated exposure of fluorescently labeled cells to illumination from lasers and high-intensity arc-discharge lamps. In their excited state, fluorescent molecules tend to react with molecular oxygen to produce free radicals that can damage subcellular components and compromise the entire cell. In addition, several reports have suggested that particular constituents of standard culture media, including the vitamin riboflavin and the amino acid tryptophan, may also contribute to adverse light-induced effects on cultured cells (Edwards, et al., 1994; Lucius, et al., 1998; Silva, et al., 1991; Spierenburg, et al., 1984). Fluorescent proteins, due to the fact that their fluorophores are buried deep within a protective polypeptide envelope, are generally not phototoxic to cells (Shaner, et al., 2005). In contrast, many of the common synthetic fluorophores, such as the MitoTracker and most nuclear stains (including Hoechst, SYTO cyanine dyes, and DRAQ5), can be highly toxic to cells when illuminated for even relatively short periods of time (Durand and Olive, 1982; Minamikawa, et al., 1999). In designing experiments, synthetic fluorophores as well as fluorescent proteins that exhibit the longest excitation wavelengths possible should be chosen in order to minimize damage to cells by short wavelength illumination. Thus, rather than creating fusion products with blue or cyan fluorescent proteins (excited by ultraviolet and blue illumination, respectively), variants that emit in the yellow, orange, and red regions of the spectrum should, when possible, be used instead.

Investigators should take care to perform the necessary control experiments when using new fluorescent protein variants, chimeric fusion protein vectors, and cell lines to ensure that cytotoxicity and phototoxicity artifacts do not obscure important biological phenomena. In some cases, lipophilic reagents induce deleterious effects that may be confused with fluorescent protein toxicity during imaging in cell lines following transient transfections. Oligomeric fluorescent proteins (discussed below) from reef corals have a far greater tendency to form aggregates (combined with poor subcellular localization) than do the monomeric jellyfish proteins, but improperly folded fusion products can occur with any variant. Recently, a fluorescent protein capable of generating reactive oxygen species (ROS) upon illumination with green light has been reported (Bulina, et al., 2006) as an effective agent for inactivation of specific proteins by chromophore-assisted light inactivation (CALI) and photodynamic therapy. Appropriately named KillerRed, this genetically encoded photosensitizer is capable of killing both bacteria and eukaryotic cells upon illumination in the microscope. Previous studies on GFP phototoxicity indicate that even through the chromophore is capable of generating singlet oxygen (Greenbaum, et al., 2000), the probe is inefficient as a photosensitizer (Rajfur, et al., 2002; Tour, et al., 2003). However, prolonged illumination of cells expressing GFP and its variants can result in physiological alterations and eventual cell death (Dixit and Cyr, 2003), a definite signal of the potential for phototoxicity in long-term imaging experiments. 
In live-cell experiments, fluorescent proteins are highly advantageous for extended imaging due to their reduced rate of photobleaching when compared to synthetic fluorophores (Rizzo and Piston, 2005a; Zhang, et al., 2002; Zumbusch and Jung, 2000). Although there is a high degree of uncorrelated variability between fluorescent proteins in terms of photostability, most variants are useful for short-term imaging (from 1 to 25 captures), while several of the more photoresistant proteins can be employed in time-lapse sequences that span periods of 24 hours or longer (in which hundreds to thousands of images are gathered). The long term stability of any particular protein, however, must be investigated for every illumination scenario (widefield, confocal, multiphoton, swept-field, etc.) because differences in photostability are often observed with the same protein when illumination is produced by an arc-discharge lamp versus a laser system. Thus, in terms of photostability, the selection of fluorescent proteins is dictated by numerous parameters, including the illumination conditions, the expression system, and the effectiveness of the imaging setup.

\section{Oligomerization}

All of the fluorescent proteins discovered to date display at least a limited degree of quaternary structure, exemplified by the weak tendency of native Aequorea victoria green fluorescent protein and its derivatives to dimerize when immobilized at high concentrations (Campbell, et al., 2002; Zacharias, et al., 2002). This tenant is also verified by the obligate dimerization necessary for solubility in fluorescent proteins isolated from Renilla sea pansies (Ward, 2006) and the strict tetramerization motif of the native yellow, orange, and red fluorescent proteins isolated in reef corals and anemones (Baird, et al., 2000; Gross, et al., 2000; Verkhusha and Lukyanov, 2004; Yarbrough, et al., 2001). Oligomerization can be a significant problem for many applications in cell biology, particularly in cases where the fluorescent protein is fused to a host protein that is targeted at a specific subcellular location. Once expressed, the formation of dimers and higher order oligomers induced by the fluorescent protein portion of the chimera can produce atypical localization, disrupt normal function, interfere with signaling cascades, or restrict the fusion product to aggregation within a specific organelle or the cytoplasm. This effect is particularly marked when the fluorescent protein is fused to partners such as actin, tubulin, or histones, which themselves participate in natural oligomer formation. Fusion products with proteins that form only weak dimers (i.e. most Aequorea victoria variants) may not exhibit aggregation or improper targeting, provided the localized concentration remains low. However, when dimeric fluorescent proteins are targeted to specific cellular compartments, such as the plasma membrane (Zacharias, et al., 2002), the localized protein concentration can, in some circumstances, become high enough to permit dimerization. This is a particular concern when conducting FRET experiments, which can yield complex data sets that are easily compromised by dimerization artifacts.

The basic strategy for overcoming oligomerization artifacts is to modify the fluorescent protein amino acid sequence to include residues that disrupt intermolecular binding, a procedure that varies in complexity depending upon the nature and origin of the protein. For many of the Aequorea protein variants, dimerization can be either significantly reduced or eliminated completely (Zhang, et al., 2002) by replacing the hydrophobic amino acid side chains in the dimer interface with positively charged residues at several key sequence positions. The three most successful mutations, in decreasing order of effectiveness, are F223R, L221K, and A206K, where the non-polar amino acids phenylalanine, leucine, and alanine are replaced by their hydrophilic relatives, arginine or lysine. In cases where close molecular associations are suspected involving a fusion protein and where quantitative FRET interactions are being investigated, it is highly recommended that Aequorea variants (CFP, GFP, YFP) should be converted into monomers using one of these point mutations (preferably A206K, Shaner, et al., 2005; Zacharias and Tsien, 2006).

Creating fluorescent protein monomers from the tetrameric coral reef and sea anemone proteins is usually far more difficult. For example, even at exceedingly low concentrations, the DsRed fluorescent protein is an obligate tetramer (Baird, et al., 2000; Vrzheshch, et al., 2000; Wiehler, et al., 2001) that cannot be dissociated without irreversible denaturation of the polypeptides (Mizuno, et al., 2001). In the tetrameric unit, each DsRed protein interacts with two adjacent neighbors, one through a hydrophobic interface and the other through a hydrophilic interface resulting in a complex assembly (Baird, et al., 2000;

Campbell, et al., 2002; Verkhusha and Lukyanov, 2004). Other Anthozoa proteins, such as the Zoanthus variants and eqFP611 (Wiedenmann, et al., 2005; Zacharias, 2002), have simpler interfaces that may prove 
easier to disrupt into monomers. The most successful approaches (Campbell, et al., 2002; Zacharias and Tsien, 2006) utilized so far to generate fluorescent protein monomers with Anthozoa species have involved repeated site-directed mutagenesis to disrupt the tetrameric interfaces, usually by substitution of hydrophilic or charged amino acids for hydrophobic and neutral moieties. Because a significant decrease in fluorescence emission quantum yield usually accompanies these genetic modifications, a second round of random mutagenesis is often necessary to rescue fluorescence (as discussed below). Another technique involves creating vectors containing two sequential coding regions separated by a short linker of nonspecific amino acids, known as "tandem dimers", from proteins that exist naturally as dimeric and tetrameric complexes. Upon expression, the fused fluorescent proteins preferentially bind to each other to form an intramolecular dimeric unit that performs as a monomer although at twice the molecular weight (and size). This method was successfully applied with HcRed by fusing two copies of the DNA sequence, separated by a short linker of four amino acids, to several subcellular localization proteins (Fradkov, et al., 2002). Tandem dimer constructs have also been developed with DsRed (Campbell, et al., 2002) and a photoconversion fluorescent protein known as Eos (Ivanchenko, et al., 2005). Other mechanisms for reducing fluorescent protein oligomerization and aggregation effects include removing several basic residues from the N-terminus (Yanushevich, et al., 2001) and simultaneous co-expression of fluorescently tagged proteins with an excess of either fusion partner alone or with a non-fluorescent mutant of the marker protein (Bulina, et al., 2003; Lauf, et al., 2001). Regardless of the specific mechanism employed to overcome fluorescent protein oligomerization, the most important point is that experimental results are not compromised by artifacts induced by the existence of quaternary structures.

\section{The Fluorescent Protein Color Palette}

A broad range of fluorescent protein genetic variants have been developed (Chudakov, et al., 2005; Shaner, et al., 2005; Verkhusha and Lukyanov, 2004) over the past several years that feature fluorescence emission spectral profiles spanning almost the entire visible light spectrum. Mutagenesis efforts in the original jellyfish green fluorescent protein have resulted in new fluorescent probes that range in color from blue to yellow (Tsien, 1998) and are some of the most widely used in vivo reporter molecules in biological research. Longer wavelength fluorescent proteins, emitting in the orange and red spectral regions, have been developed from the marine anemone Discosoma striata and reef corals belonging to the class Anthozoa (Matz, et al., 1999). Still other species have been mined to produce similar proteins having cyan, green, yellow, orange, red, and far-red fluorescence emission (Chudakov, et al., 2005; Shaner, et al., 2005). Developmental research efforts are ongoing to improve the brightness and stability of fluorescent proteins, thus improving their overall usefulness.

Essential to the understanding of spectral diversity in the wide range of fluorescent proteins discovered thus far are structural investigations of the stereochemical nature of the fluorophore and the effects of its surrounding environment on fluorescent properties. Aside from the jellyfish proteins, there appears to be a high degree of variation in the fluorophores of red-shifted fluorescent proteins (Shkrob, et al., 2005). Even through the DsRed fluorophore configuration, termed planar cis, appears to be the predominant structure in most proteins that emit in the orange and red regions, there are at least two additional motifs, planar trans and non-planar trans, which have been elucidated through x-ray diffraction studies. A planar trans motif is found in the red fluorescent protein eqFP611, isolated from the sea anemone Entacmaea quadricolor (Peterson, et al., 2003), which displays one of the largest Stokes shifts and red-shifted emission wavelength profiles of any naturally occurring Anthozoan fluorescent protein. In contrast, the non-planar trans conformation is characteristic of the non-fluorescent chromoprotein Rtms5 from Montipora efflorescens (Beddoe, et al., 2003). As further studies into the complex characteristics of fluorescent protein chromophores yield clues about the structure-function relationship with the polypeptide backbone, the task of genetically engineering more finely-tuned color variants and broadening the spectral range of useful proteins will undoubtedly become easier.

\section{Blue Fluorescent Proteins}

Fluorescent proteins emitting in the blue region of the visible light spectrum (approximately 440 to $470 \mathrm{~nm}$ ) were first obtained from site-directed mutagenesis efforts targeted at the tyrosine amino acid residue at position 66 in the GFP chromophore. Conversion of this residue to histidine (Y66H) produces a 
blue fluorescent protein (BFP) that exhibits a broad absorption band in the ultraviolet centered close to 380 $\mathrm{nm}$ and an emission maximum at $448 \mathrm{~nm}$ (Cubitt, et al., 1999; Heim, et al., 1994). The original protein exhibited only about $15-20 \%$ of the parent GFP brightness value due to a low quantum yield and required additional secondary mutations to increase its folding efficiency and expression levels. Subsequent investigations and several additional mutations led to an enhanced BFP version that is still only $25 \%$ as bright as the enhanced green variant (Patterson, et al., 1997; Patterson, et al., 2001; Yang, et al., 1998) and displays limited photostability compared to many other fluorescent proteins.

The primary motivation for developing blue fluorescent proteins in the mid-to-late 1990s was the keen interest in creating matched pairs for fluorescence resonance energy transfer (FRET) experiments and multicolor labeling (Tsien, 1998). Because the spectral characteristics (fluorescence emission profile) of BFP are readily distinguishable from EGFP, this protein combination was one of the first utilized for multicolor imaging (Heim and Tsien, 1996; Rizzuto, et al., 1996). Blue fluorescent protein also has the distinction of being incorporated into the first genetically encoded biosensor along with an enhanced GFP variant to demonstrate FRET through linkage of the two fluorescent proteins via an intervening proteasesensitive spacer (Heim and Tsien, 1996; Mitra, et al., 1996). The broad emission peak of BFP overlaps to a significant extent with the excitation spectrum of red-shifted GFP variants to yield a Förster distance of 4.1 (Patterson, et al., 2000), a reasonable value for measuring FRET. Blue fluorescent protein has also been coupled with several GFP derivatives into biosensors designed to monitor transcription factor dimerization (Periasamy and Day, 1998), calcium (Miyawaki, et al., 1997; Romoser, et al., 1997), and apoptosis (Rhem, et al., 2002).

Aside from the limited brightness levels and rapid photobleaching, blue fluorescent proteins also suffer from the fact that they must be excited with ultraviolet light, which is phototoxic to mammalian cells, even in limited doses (Khodjakov and Rieder, 2006; Potter, 1996; Stevens and Allan, 2003). Furthermore, working in this spectral region is often hampered by autofluorescence and high absorption levels by cells and tissues, as well as light scattering. Microscopes operating in the ultraviolet also require specialized light sources, optics, and filter combinations that further complicate imaging (Rizzo and Piston, 2005a). For all of the reasons listed above, the quest for more efficient blue fluorescent proteins has only been pursued by a few research groups. Investigations of mutagenesis using non-natural amino acids positioned in and around the chromophore have led to several blue-shifted "artificial" fluorescent protein variants that may find utility in several biological and photophysical applications (Kajihara, et al., 2005).

\section{Cyan Fluorescent Proteins}

The first fluorescent protein emitting in the bluish-green cyan spectral region (CFP; ranging from approximately 470 to $500 \mathrm{~nm}$ ) was discovered simultaneously with BFP during mutagenesis studies (Heim, et al., 1994) that converted the tyrosine residue in the GFP chromophore to tryptophan (Y66W). This single mutation yielded a chromophore that displays an absorption maximum at $436 \mathrm{~nm}$ with a very broad fluorescence emission spectral profile centered at $485 \mathrm{~nm}$. Subsequent refinements, including the F64L maturation improvement and S65T (discussed in Green Fluorescent Proteins), resulted in the production of an enhanced version (ECFP) with greater brightness and photostability (Cubitt, et al., 1995; Patterson, et al., 2001). Even these modifications failed, however, to increase the brightness level of ECFP beyond $40 \%$ of that shown by EGFP. Other than providing an additional hue for multicolor imaging, initially the most promising aspect of ECFP was the potential for utility as a biosensor FRET partner with yellow fluorescent proteins in widefield and confocal microscopy using blue-violet filter sets or the 457-nm spectral line of an argon-ion laser (Miyawaki, et al., 1997; Tsien, 1998). Greater photostability compared to EBFP also positions ECFP as a more useful protein for time-lapse imaging in localization and dynamics studies.

By far the most successful applications using ECFP have taken advantage of the ability of this variant to be coupled with yellow fluorescent protein (YFP) and derivatives to generate FRET biosensors capable of monitoring a wide spectrum of intracellular processes (reviewed in Miyawaki, 2003; Meyer and Teruel, 2003; Zaccolo, 2004; Zhang, et al., 2002). Although a host of FRET investigations have been performed using the ECFP-EYFP pair, the experimental results are often problematic due to limitations in the fluorophore properties that restrict measurements to a small dynamic range (Rizzo and Piston, 2005a). A majority of these biosensors exhibit a typical overall ratio change of 10 to $30 \%$ during FRET analysis, 
with a few exceptions (Ting, et al., 2001; Zhang, et al., 2001; Rizzo, et al., 2002). Such a low level of contrast presents difficulties with modern digital microscopy due to a noise level that can approach $10 \%$ of the signal at low imaging intensities (Swedlow, et al., 2002). These biosensors are further complicated by the potential to form dimers when constrained with tight two-dimensional spatial restrictions such as those that exist in membranes (Ward, 2006). To overcome the artifacts associated with dimerization, hydrophobic residues at the dimer interface were replaced with positively charged amino acids in both ECFP and EYFP to produce true monomeric variants (mCFP and mYFP), which achieve a far higher level of efficiency in FRET experiments (Zacharias, et al., 2002). The critical mutation is the substitution of lysine for alanine at position 206 (A206K, as discussed above), which can be applied to virtually any Aequorea victoria variant in order to generate a true monomer capable of superior performance in applications such as localization, FRET, and protein dynamics.

\section{Subcellular Localization of Fluorescent Protein Chimeras}
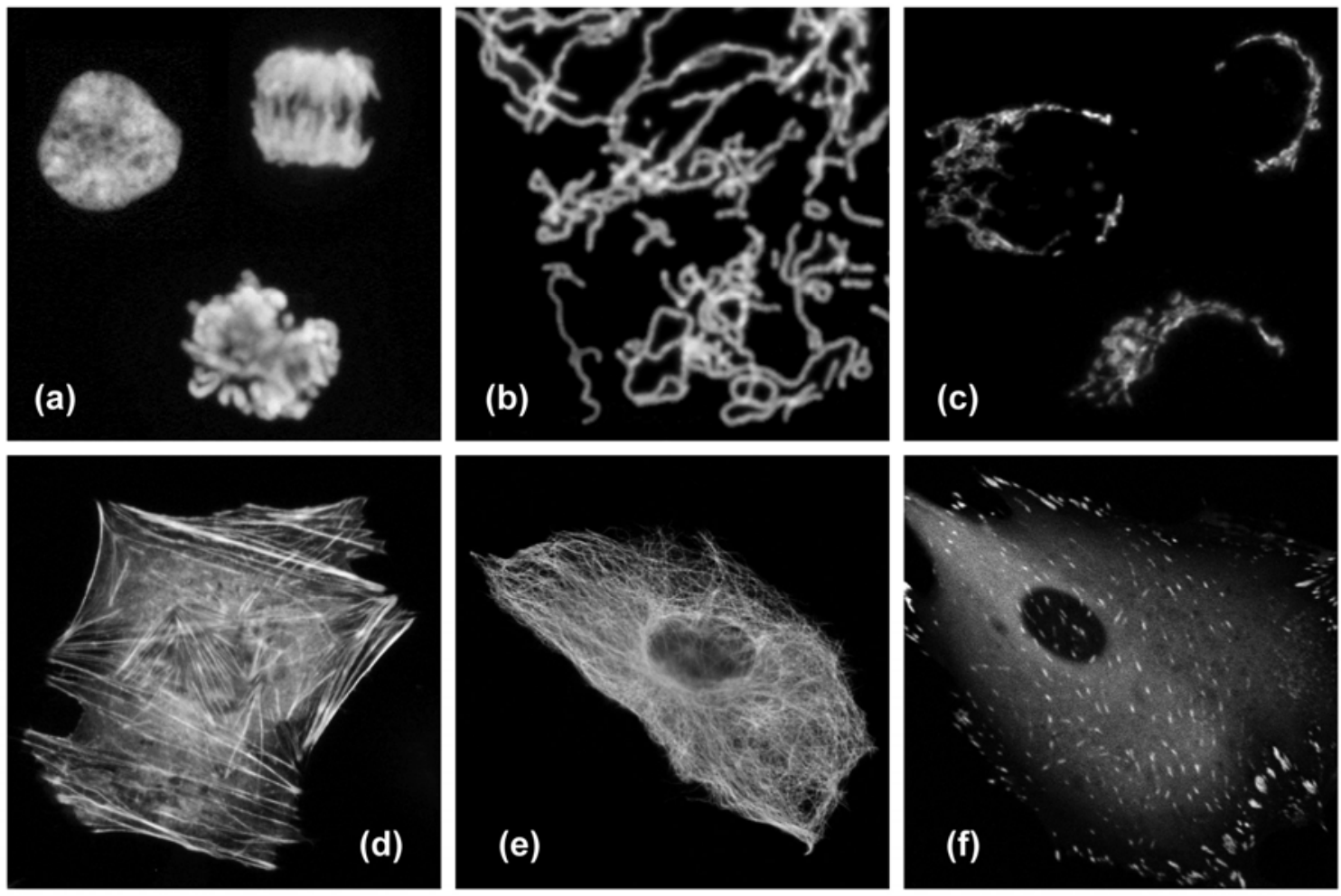

Figure 3. Localization of fluorescent protein fusion tags to specific subcellular compartments. (a) The chimera of mPlum and histone H2B localizes to the nucleus and can be utilized to observe chromosomes during cell division. (b) The monomeric red fluorescent protein mCherry highlights mitochondria when fused to the targeting sequence from subunit VIII of human cytochrome c oxidase. (c) A fusion between EYFP and the N-terminal 81 amino acids of human $\beta$-1,4-galactosyltransferase localizes the yellow fluorescent marker to the Golgi apparatus. (d) The actin cytoskeleton becomes fluorescent when Cerulean fluorescent protein is fused to the gene for human $\beta$-actin. (e) A EGFP-tubulin fusion vector marks the microtubule network in COS-7 cells. (f) Focal adhesions become fluorescent with a chimera of mKusabira Orange fluorescent protein and vinculin.

Despite the host of improvements in CFP and the large database of experiments performed with this variant, continued investigation has produced additional useful fluorescent proteins in the cyan spectral class. Among the improved cyan fluorescent proteins that have recently been introduced, CyPet and an enhanced cyan variant termed Cerulean show the most promise (Nguyen and Daugherty, 2005; Rizzo, et al., 2004) as fusion tags, FRET biosensors, and for multicolor imaging (see Figures 3 and 4). The Cerulean 
fluorescent probe (named for the sky-blue color) was rationally engineered by site-directed mutagenesis of enhanced cyan fluorescent protein to yield a higher extinction coefficient, improved quantum yield, and a fluorescence lifetime decay having a single exponential component (useful in lifetime decay measurements of FRET; Wallrabe and Periasamy, 2005). Cerulean is at least 2-fold brighter than enhanced cyan fluorescent protein and has been demonstrated to significantly increase contrast as well as the signal-tonoise ratio when coupled with yellow-emitting fluorescent proteins, such as Venus (see below), in FRET investigations. In addition to site-directed mutations designed to improve folding and brightness (Rizzo, et al., 2004), random mutagenesis was conducted on pre-Cerulean variants to further increase the molar extinction coefficient, and the optimized protein has been "monomerized" to enhance its utility in fusions (Rizzo and Piston, 2005b). The abundance of advantageous features afforded by Cerulean render this protein the most useful all-purpose cyan derivative.

The cyan fluorescent protein variant named CyPet (from the acronym: Cyan fluorescent Protein for energy transfer) was derived through a quantitative and unique strategy utilizing fluorescence-activated cell sorting (FACS; Boeck, 2001) to optimize the cyan and yellow pairing for FRET (Nguyen and Daugherty, 2005). Libraries were screened for FRET efficiency and the best clones were subjected to several evolutionary cycles consisting of random mutagenesis and synthetic DNA shuffling. A total of seven mutations resulted in the production of the CyPet protein, which features absorption and emission maxima positioned at 435 and $477 \mathrm{~nm}$, respectively. CyPet is about half as bright as EGFP and two-thirds as bright as Cerulean, but expresses relatively poorly at $37^{\circ} \mathrm{C}$ (Shaner, et al., 2005). However, when paired with its FACS-optimized partner, YPet, in FRET biosensors, this cyan variant exhibits a dynamic range that is more than six times higher than CFP-YFP (Nguyen and Daugherty, 2005), dramatically improving contrast and potentially leading to far more sensitive detection of subtle intracellular processes. CyPet has a more blue-shifted and narrower fluorescence emission peak than mCFP, which greatly increases its utility in multicolor imaging, and is the most photostable cyan fluorescent protein of the weakly dimeric and monomeric versions currently available.

Several potentially useful cyan proteins have been isolated in Anthozoan species. Derived from the reef coral Anemonia majano, the AmCyan1 fluorescent protein (Matz, et al., 1999), which is now commercially available (Clontech), has been optimized with human codons for enhanced expression in mammalian cell systems (Richards, et al., 2002). Originally named amFP486 (am, Anemonia majano; FP, fluorescent protein; 486 emission maximum) in accordance with a nomenclature scheme (Matz, et al., 1999) devised to simplify the discussion of myriad Anthozoan proteins, this variant exhibits a similar brightness level, but a significantly better resistance to photobleaching than CFP. The absorption maximum of AmCyan1 occurs at $458 \mathrm{~nm}$ while the fluorescence emission peak resides at $489 \mathrm{~nm}$. Note that both peaks are shifted to longer wavelengths by 19 and $13 \mathrm{~nm}$, respectively, compared to ECFP. On the downside, similar to most of the other reef coral proteins, the probe has a tendency to form tetramers, which will significantly complicate attempts to employ this protein as a fusion tag or a FRET biosensor.

First isolated by Miyawaki and associates from an Acropara stony coral species (Karasawa, et al., 2004), the cyan-emitting Midoriishi-Cyan (abbreviated MiCy) probe was originally designed as the donor in a new FRET combination with the monomeric Kusabira Orange fluorescent protein to generate a biosensor probe with high spectral overlap (Förster distance of 5.3; mKO is discussed in the section on Orange Fluorescent Proteins). This protein features the longest absorption and emission wavelength profiles (472 and $495 \mathrm{~nm}$, respectively) reported for any probe in the cyan spectral class. The high molar extinction coefficient and quantum yield exhibited by MiCy render the protein of equal brightness to Cerulean, although the spectra are far more sensitive to $\mathrm{pH}$. Also similar to Cerulean, MiCy features a single exponential lifetime decay component with a time constant of $3.4 \mathrm{~ns}$, which should be useful for measurements of FRET in combination with fluorescence lifetime imaging microscopy (FLIM). An unusual feature of MiCy is that it forms a homodimeric complex similar to the GFP variant isolated from the bioluminescent sea pansy, Renilla reniformis (Ward and Cormier, 1979), rather than the obligate tetramer observed in most coral reef species. Although the dimerization motif may be a problem in some fusion proteins, it should be far easier than a tetramer to mutate MiCy into a true monomer. 


\section{Green Fluorescent Proteins}

The original green fluorescent protein isolated from Aequorea victoria (termed wild-type) has been the principal subject of numerous investigations (Tsien, 1998), but is not useful in a majority of the practical applications involving fluorescent proteins due to the bimodal absorption band (395 and $475 \mathrm{~nm}$ peaks), which is hampered by relatively low extinction coefficients and an absorption maximum in the ultraviolet part of the spectrum. Shortly after GFP was demonstrated to be a useful marker for gene expression (Chalfie, et al., 1994), a point mutation altering the serine residue at position 65 in the chromophore into threonine (S65T) produced a new version of the protein having a well defined absorption profile with a single peak at $484 \mathrm{~nm}$ (Heim, et al., 1995). This mutation is featured in the most popular variant of green fluorescent protein, termed enhanced green fluorescent protein (EGFP), which can be imaged using commonly available filter sets designed for fluorescein (FITC) and is among the brightest and most photostable of the Aequorea victoria fluorescent proteins (Shaner, et al., 2005). These features have rendered enhanced green fluorescent protein one of the most popular probes and the best choice for most single-label fluorescent protein experiments. As previously discussed, EGFP was coupled with blue fluorescent proteins as a FRET acceptor in early biosensor investigations, but has subsequently been largely replaced by yellow, orange, and red variants. The only drawbacks to the use of EGFP as a fusion tag are a slight sensitivity to $\mathrm{pH}$ and a weak tendency to dimerize.

In addition to EGFP, several other green-emitting variants (in the range of approximately 500 to $525 \mathrm{~nm}$ ) are currently being utilized in live-cell imaging (Rizzo and Piston, 2005a). Among the best of these in terms of photostability and brightness may be the Emerald variant (Cubitt, et al., 1999), but lack of a commercial source (until recently) has limited its use. Emerald contains the S65T mutation featured in EGFP, but also has four additional point mutations that improve folding, expression at $37^{\circ} \mathrm{C}$, and brightness. Although Emerald is far more efficient than EGFP in folding and developing fluorescence in mammalian cells, it has a fast photobleaching component that might affect quantitative imaging in some environments. One of the most interesting derivatives of wtGFP is Sapphire (Zapata-Hommer and Griesbeck, 2003), which contains a critical mutation of isoleucine for threonine at position 203 (T203I), abolishing the absorption peak at $475 \mathrm{~nm}$. The result is a protein having an absorption maximum at $399 \mathrm{~nm}$ with emission in the green spectral region $(511 \mathrm{~nm})$ to yield a surprisingly large Stokes shift of over 100 $\mathrm{nm}$. Several Sapphire variants have been developed to improve folding, including a probe known as TSapphire (for Turbo), and derivatives featuring circular permutations are available that might be useful for altering the orientation geometry with fusion partners. Due to the significant separation between absorption and emission peaks, the greatest potential for the Sapphire proteins is their pairing with orange and red derivatives as FRET donors.

A wide variety of additional fluorescent proteins emitting in the green spectral region have been isolated from other sources, including different Aequorea species, copepods, and coral reefs. One of the most promising of these probes, derived by random mutagenesis of a colorless protein isolated from Aequorea coerulescens, is known as aceGFP (Gurskaya, et al., 2003). The conversion of glutamic acid into glycine at position 222 (E222G) transformed the wild-type protein into a highly fluorescent species with a relatively symmetrical pair of spectral profiles having an absorption maximum at $480 \mathrm{~nm}$ and an emission peak at $505 \mathrm{~nm}$. The high molar extinction coefficient and quantum yield of aceGFP combine to produce a brightness level similar to that displayed by EGFP. Demonstrated to exist as a monomer by electrophoresis and gel filtration, this protein is commercially available from several sources (Clontech and Evrogen, as AcGFP1 and AceGFP) with human-optimized codon replacements. Proper localization of fusion products targeted at specific subcellular components and organelles (such as filamentous actin, the Golgi, nucleus, mitochondria, etc.; see Figure 3) indicates that aceGFP is quite useful as a marker and could have potential for partnership with red-emitting proteins in a novel FRET combination. However, the photostability characteristics of aceGFP remain unknown, and there are no clear advantages to the use of this protein over the more common EGFP and Emerald variants.

Several closely related GFP-like proteins have been isolated from an assortment of copepod aquatic crustacean species. The brightest of these probes, originally referred to as ppluGFP2 (Shagin, et al., 2004), have been made commercially available (Evrogen) under the names CopGFP and TurboGFP (an enhanced variant). CopGFP is efficiently excited using an argon-ion laser or FITC filter set (absorption 
maximum at $482 \mathrm{~nm}$ ) and produces green fluorescence at $502 \mathrm{~nm}$ with a brightness value approximately $30 \%$ higher than EGFP and a much greater resistance to changes in $\mathrm{pH}$. Reported to be a monomer in dilute solution, CopGFP matures significantly faster than EGFP and is ideal for applications as a fusion partner targeted at expression in subcellular regions of low $\mathrm{pH}$. Limitations of this probe include the inability to isolate stable cell lines and the formation of aggregates in long-term cultures. An improved version, TurboGFP, derived from site-directed and random mutagenesis, retains the fast maturation kinetics of the parent protein with a slight loss in brightness and substantially lower resistance to acidic environments. Despite the improved folding kinetics and excellent optical properties of these proteins, however, photostability data have not been reported and no compelling evidence exists to demonstrate a significant benefit over the application of the extensively studied original GFP derivatives.

Green fluorescent proteins have also been mined from reef corals and several of these are commercially available. A brightly fluorescent protein termed Azami Green (Karasawa, et al., 2003), bearing only a surprisingly scant (less than 6\%) sequence homology to EGFP, was isolated from the stony coral Galaxeidae and has been demonstrated to mature rapidly during expression in mammalian cell lines. Likewise, one of the original Anthozoa coral reef proteins from Zoanthus reported by Matz and coworkers (Matz, et al., 1999) has also been transformed into a commercial product (Clontech) under the tradename ZsGreen. The probes have absorption maxima at 492 and $496 \mathrm{~nm}$ and emission peaks at 505 and $506 \mathrm{~nm}$, respectively, readily allowing visualization and imaging with standard lasers and filter combinations in confocal and widefield microscopy. However, similar to most of the other proteins isolated in corals, Azami Green and ZsGreen both exist as tetramers in the natural state, which significantly interferes with their use as fusion partners and as a FRET donor or acceptor in biosensors. To overcome the oligomerization problem, site-directed and random mutagenesis efforts were successful in creating a monomeric version of Azami Green, but this type of effort has not been reported for ZsGreen although the protein has been re-engineered with human codons to optimize expression (resulting in a variant termed ZsGreen1). Because reliable photostability data is lacking, it is unclear whether either of these proteins will outperform EGFP in long-term imaging experiments.

The sea pansy, an Anthozoa soft coral, is the source of several green fluorescent proteins that have been characterized in detail and are now commercially available (Cormier and Eckroade, 1962; Morin and Hastings, 1971, 1977; Miyawaki, 2002). A protein isolated from Renilla reniformis that exhibits properties similar to EGFP is the best characterized of the probes in this class. Having absorption and emission maxima at 485 and $508 \mathrm{~nm}$, respectively, in addition to a similar sensitivity to $\mathrm{pH}$, the Renilla protein would be an excellent substitute for EGFP were it not for the fact that it is an obligate dimer (Ward, 2006). Aside from the oligomerization problem, Renilla GFPs may be useful in many applications and have been expressed in a wide variety of organisms, including bacteria, fungi, and mammalian cells. Versions with human codon sequences are available from the manufacturers, as are derivatives optimized for expression in other species. There is a general lack of reliable data concerning extinction coefficients, quantum yields, and photostability for the commercial Renilla proteins, so valid comparisons to EGFP in terms of brightness and photobleaching are not possible.

\section{Yellow Fluorescent Proteins}

Yellow fluorescent proteins, as a spectral class, are among the most versatile genetically-encoded probes yet developed. Ranging in emission wavelength maxima from approximately 525 to $555 \mathrm{~nm}$, those proteins residing in the shorter wavelength region actually appear green, rather than yellow, when viewed in a widefield fluorescence microscope. The first member in what has become a rather large family of probes was rationally engineered after the high resolution crystal structure of green fluorescent protein revealed that threonine residue 203 (Thr203) was positioned near the chromophore and potentially able to alter the spectral characteristics upon substitution (Ormö, et al., 1996; Rizzo and Piston, 2005a). Mutations of this aliphatic amino acid to several aromatic moieties were introduced in order to induce $\pi$-orbital stacking and attempt stabilization of the excited state dipole moment of the chromophore. The most successful mutant proved to be tyrosine (T203Y; termed mutant 10C, the original YFP), which resulted in almost a 20-nm shift to longer wavelengths for both the excitation and emission spectra (Ormö, et al., 1996; Tsien, 1998; Wachter, et al., 1998). Several YFP variants were initially constructed in attempts to maximize brightness as well as to increase the speed of maturation and optimize expression at $37^{\circ} \mathrm{C}$ 
(Cubitt, et al., 1999; Tsien, 1998). One of these variants, named Topaz, has been of service in fusion tag localization, intracellular signaling, and FRET investigations (Cornea and Conn, 2002; Sturman, et al., 2006; Tavaré, et al., 2001).

In an effort to improve the performance of FRET biosensors, further sequence refinements led to the development of the enhanced yellow fluorescent protein (EYFP), which is one of the brightest and most widely utilized fluorescent proteins (Miyawaki, 2005; Miyawaki, et al., 1999; Shaner, et al., 2005). EYFP was constructed from the original yellow variant by introduction of lysine to position 69 in place of glutamine (Q69K). The high brightness level and fluorescence emission spectrum wavelength profile of EYFP combine to make this probe an excellent candidate for multicolor imaging experiments in fluorescence microscopy, although maturation is slower, especially in organelles. Enhanced yellow fluorescent protein has also been widely employed as an acceptor for energy transfer experiments when paired with enhanced cyan fluorescent protein. However, all of the original yellow fluorescent protein variants present some problems in that they are very sensitive to acidic $\mathrm{pH}$ and lose approximately $50 \%$ of their fluorescence at $\mathrm{pH}$ 6.5. In addition, most of the Aequorea-derived proteins in this class have also been demonstrated to have significant sensitivity to chloride ions, undergo weak dimerization, exhibit relatively poor expression at $37^{\circ} \mathrm{C}$, and to photobleach much more readily than many of the green fluorescent proteins. Several investigations have taken advantage of the environmental sensitivity of YFP to measure cytosolic pH (Llopis, et al., 1998), chloride ion concentrations (Jayaraman, et al., 2000; Kuner and Augustine, 2000), and FRET efficiency (Llopis, et al., 2000). The A206K mutation, substituting the charged amino acid lysine for alanine at the hydrophobic dimer interface, has been applied to YFP (Zacharias, et al., 2002) in order to generate a true monomer.

Continued genetic development of the YFP family (Griesbeck, et al., 2001) led to the discovery that a single point mutation near the chromophore, the substitution of methionine for glutamine at position 69 (Q69M), dramatically increases the acid stability of the protein and reduces the chloride sensitivity. Named Citrine in recognition of the yellow color and acid resistance, this variant also expresses at a much higher level in mammalian cell culture (especially when targeted to acidic organelles) and demonstrates almost twice the photostability of many previous yellow fluorescent proteins. Citrine features absorption and fluorescence emission maxima at 516 and $529 \mathrm{~nm}$, respectively, and is 75\% brighter than EGFP, although much less photostable. FRET biosensors constructed with Citrine demonstrate better performance than sister analogs containing EYFP, and the probe is a better choice for multicolor experiments in general. Although Citrine exists as a weak dimer in solution, the protein can be converted into a monomer with the A206K mutation (Shaner, et al., 2005).

Introduction of yet another novel point mutation into Aequorea-derived YFP, the substitution of leucine for phenylalanine at position 46 (F46L), produced a variant that was named Venus in honor of the brightest star in the night sky (Nagai, et al., 2002). This mutation, which was discovered during random mutagenesis experiments with circularly permuted GFP derivatives, dramatically accelerates oxidation of the chromophore, the rate-limiting step in fluorescent protein maturation. Additional mutations were also introduced in order to increase the tolerance of Venus to acidic environments and to reduce the sensitivity to chloride. The absorption and emission spectral peaks (515 and $528 \mathrm{~nm}$, respectively) of Venus are shifted to longer wavelengths by a single nanometer compared to EYFP, but the brightness level is retained. Unfortunately, the photostability of Venus is only about 25\% that of EYFP, a significant problem for long-term imaging experiments. Venus has been demonstrated to perform well in intracellular organelle fusion protein $\mathrm{pH}$ assays (Tsuboi and Rutter, 2003), for localization studies in yeast (Sheff and Thorn, 2004), in bimolecular fluorescence complementation analysis (Shyu, et al., 2006), and holds significant potential as an acceptor in FRET biosensors (Sheridan and Hughes, 2004). Genetic conversion of the weakly dimeric Venus into a monomer using the A206K mutation will further expand the use of this probe in biological investigations (Rizzo and Piston, 2005b).

During the same fluorescence-activated cell sorting investigation that led to the generation of the cyan fluorescent protein CyPet (Nguyen and Daugherty, 2005), the evolutionary optimized complementary FRET acceptor, termed YPet, was also obtained from Venus and YFP variants. Named after its proficiency in FRET (YFP for energy transfer), YPet is the brightest yellow fluorescent protein variant yet developed and demonstrates very good photostability. The resistance to acidic environments afforded by YPet is 
superior to Venus and other YFP derivatives, which will enhance the utility of this probe in biosensor combinations targeted at acidic organelles. However, although the optimized CyPet-YPet combination should be the preferred starting point in the development of new FRET biosensors, the utility of this pair has not yet been tested in widespread practice (Shaner, et al., 2005). Likewise, the suitability of CyPet and YPet in fusion tags for localization experiments, bimolecular complementation analysis, and other routine fluorescent protein assays has yet to be established. Both proteins exist in solution as weak dimers, but presumably can be converted to true monomers using the A206K mutation that has worked so well with other Aequorea variants.

Although the potential for new discoveries of yellow and green fluorescent proteins in Hydrozoan species other than Aequorea victoria is significant, only one candidate has surfaced so far. Isolated from the Phialidium jellyfish, a protein termed phiYFP (Shagin, et al., 2004) is reported to demonstrate very bright yellow fluorescence (absorption and emission at 525 and $537 \mathrm{~nm}$, respectively) and to be useful for $\mathrm{N}$-terminal fusion tags. An extraordinary feature of phiYFP is that the naturally occurring protein contains the same mutation at position 64 (leucine) that was introduced by Venus to increase the folding efficiency (Nagai, et al., 2002). The probe also naturally contains tyrosine at position 203 (Ormö, et al., 1996), another site-directed modification of the native GFP that resulted in yellow fluorescence. This remarkable discovery of a natural similarity between the structure of phiYFP and genetically modified Aequorea proteins is a testament to the efficacy of protein engineering efforts directed at GFP to adjust the spectral properties. The phiYFP protein has been optimized by random mutagenesis to produce a monomeric version without compromising the spectral properties.

Site-directed and random mutagenesis of a monomeric Anthozoa fluorescent protein (mRFP1) from Discosoma have resulted in the creation of two monomeric coral reef derivatives with spectral properties falling in the range of Aequorea yellow fluorescent proteins (Shaner, et al., 2004). Named after similarly colored fruits, mHoneydew and mBanana both emit fluorescence in the yellow spectral region. The broad absorption band of mHoneydew (peaks at 487 and $504 \mathrm{~nm}$ ) enables effective excitation with an argon-ion laser or standard FITC filter combination. However, the similarly broad emission spectrum (peaks at 537 and $562 \mathrm{~nm}$ ) tails into the near-infrared, hampering the use of this protein in multicolor experiments. In addition, a low extinction coefficient and quantum yield render mHoneydew the dimmest member of the monomeric yellow fluorescent protein cadre. The mBanana variant is only twice as bright as mHoneydew, but features much narrower excitation and emission spectra (peaks at 540 and $553 \mathrm{~nm}$, respectively). Because both proteins exhibit relatively poor photostability, and mBanana is highly $\mathrm{pH}$ sensitive, neither will probably find great utility in imaging experiments. Perhaps the most promising aspect of these probes is that the mere existence of mHoneydew (a cyan-type Y67W mutant) demonstrates that the tryptophan-based chromophore of CFP can undergo a further maturation into a longer-wavelength emitting species (Shaner, et al., 2004).

ZsYellow (originally referred to as zFP538) is a yellow fluorescent protein that was discovered in the Anthozoan button polyp Zoanthus during a search in reef corals for naturally occurring GFP analogs emitting fluorescence in longer wavelength regions (Gurskaya, et al., 2001; Matz, et al., 1999; Yanushevich, et al., 2002). One of the most unique features of the ZsYellow fluorescence emission spectrum is that the peak (538 nm) occurs almost midway between those of EGFP $(508 \mathrm{~nm})$ and DsRed (583 nm), presenting an opportunity to investigate proteins emitting fluorescence in the yellow portion of the visible light spectrum. The ZsYellow fluorescent protein chromophore features a novel three-ring system and peptide backbone cleavage due to the substitution of lysine for serine as the first amino acid residue in the chromophore tripeptide sequence (Remington, et al., 2005; Zagranichny, et al., 2004). As a result of the unique chromophore motif, the degree of conjugation observed in ZsYellow is intermediate between that observed with EGFP and DsRed (one double bond more than EGFP, and one less than DsRed), which accounts for the positioning of emission wavelengths in the yellow region. ZsYellow exhibits a marked tendency to form tetramers when expressed in vivo, hampering the use of this protein as a fusion partner for localization investigations. Furthermore, the reduced brightness level of ZsYellow when compared to enhanced green fluorescent protein (25\% of EGFP) also limits the utility of this reporter in fluorescence microscopy (the human codon-optimized version is commercially available as ZsYellow1). The unique emission spectral profile of ZsYellow, however, should encourage the search for genetic modifications that alleviate the tendency to form tetramers while simultaneously increasing the quantum 
yield and extinction coefficient, an effort that could ultimately yield a high-performance monomeric yellow fluorescent protein.

\section{Orange Fluorescent Proteins}

In contrast to the relatively large number of fluorescent proteins engineered in the cyan, green, and yellow spectral classes, only three probes have been developed so far in the orange portion of the spectrum (ranging from approximately 555 to $580 \mathrm{~nm}$ ). Even so, all three of the existing orange fluorescent proteins, which were isolated from coral reef species, have the potential to be useful in a variety of imaging scenarios. Perhaps the most versatile of these is monomeric Kusabira Orange (Karasawa, et al., 2004), a protein originally derived as a tetramer from the mushroom coral Fungia concinna (known in Japanese as Kusabira-Ishi). Kusabira Orange was engineered by site-specific mutagenesis from a cDNA clone of the coral by adding ten amino acids to the N-terminus. The resulting protein has an absorption maximum at $548 \mathrm{~nm}$ (ideal for excitation with a 543-nm laser) and emits bright orange fluorescence at $561 \mathrm{~nm}$. A monomeric version of Kusabira Orange (abbreviated mKO) was created using a strategy similar to that employed for DsRed to create mRFP1 (discussed in the section on red fluorescent proteins) by introducing over 20 mutations through site-directed and random mutagenesis. The monomer exhibits similar spectral properties to the tetramer and has a brightness value similar to EGFP, but is slightly more sensitive to acidic environments. The photostability of this probe, however, is the best of any fluorescent protein in all of the spectral classes, making $\mathrm{mKO}$ an excellent choice for long-term imaging experiments. Furthermore, the emission spectral profile is sufficiently well separated from cyan fluorescent proteins to increase the FRET efficiency in biosensors incorporating $\mathrm{mKO}$, and the probe is useful in multicolor investigations (see Figure 4) with a combination of cyan, green, yellow, and red fluorescent proteins.

The mRFP1 derivative, mOrange (Shaner, et al., 2004), was derived after four rounds of directed evolution to yield a probe absorbing at $548 \mathrm{~nm}$ and emitting orange fluorescence at $562 \mathrm{~nm}$. The mOrange variant is slightly brighter than mKusabira Orange, but has less than $10 \%$ the photostability, thus severely compromising its application for experiments that require repeated imaging. However, mOrange remains the brightest protein in the orange spectral class and is still an excellent choice where intensity is more critical than long-term photostability. In addition, combined with the green-emitting T-Sapphire, mOrange is a suitable alternative to CFP-YFP proteins as a FRET pair to generate longer wavelength biosensors, and can be coupled with fluorescent proteins in other spectral regions for multicolor investigations. A novel orange fluorescent protein isolated from the Cerianthus tube anemone (Ip, et al., 2004) is commercially available (tradename cOFP; Stratagene) and has spectral properties that are similar to mOrange and mKusabira Orange, but like the other anemone proteins isolated to date, exists in solution as a tetramer. The brightness and photostability of cOFP have not been reported so this protein cannot be directly compared to other orange fluorescent proteins, and its utility will be further limited until it can be converted into a monomer.

\section{Red Fluorescent Proteins}

The quest for a well-behaved red-emitting fluorescent protein has long been the "holy grail" of live-cell imaging, primarily due to the requirement for probes in this spectral region in multicolor imaging experiments as well as the fact that longer excitation wavelengths generate less phototoxicity and can probe deeper into biological tissue. As an added convenience, most of the proteins in the red region of the visible spectrum can be imaged with the common TRITC and Texas Red widefield fluorescence filter sets, as well as cheap helium-neon (543, 561, 594, and $633 \mathrm{~nm}$ ) lasers in confocal microscopy. After five years of unsuccessful mutagenesis efforts in the Aequorea GFP-derived proteins (Tsien, 1998), the first real breakthrough occurred with the discovery of potentially fluorescent chromoproteins in non-bioluminescent Anthozoa coral species (Matz, et al., 1999). To date, a wide spectrum of potentially useful red fluorescent proteins has been reported (spanning the emission wavelength range of 580 to $630 \mathrm{~nm}$ ), many of which still suffer from some degree of the obligatory quaternary structure bestowed by their species of origin (Chudakov, et al., 2005; Miyawaki, 2005; Shaner, et al., 2005; Verkhusha and Lukyanov, 2004). Unlike the jellyfish proteins, most of the native and genetically engineered variants of coral reef proteins mature very efficiently at $37^{\circ} \mathrm{C}$, presumably due to differing water temperatures of their respective habitats (Chudakov, et al., 2005; Miyawaki, et al., 2005). 


\begin{tabular}{|c|c|c|c|c|c|c|c|}
\hline $\begin{array}{c}\text { Protein } \\
\text { (Acronym) }\end{array}$ & $\begin{array}{c}\mathbf{E x} \\
(\mathrm{nm})\end{array}$ & $\begin{array}{c}\mathbf{E m} \\
(\mathbf{n m})\end{array}$ & $\begin{array}{c}\text { EC } \\
\times 10^{-3}\end{array}$ & QY & $\begin{array}{c}\text { in vivo } \\
\text { Structure }\end{array}$ & $\begin{array}{c}\text { Relative } \\
\text { Brightness } \\
\text { (\% of EGFP) }\end{array}$ & References \\
\hline \multicolumn{8}{|c|}{ Blue Fluorescent Proteins } \\
\hline EBFP & 383 & 445 & 29.0 & 0.31 & Monomer* & 27 & Patterson, et al., 1997 \\
\hline Sapphire & 399 & 511 & 29.0 & 0.64 & Monomer* & 55 & Zapata-Hommer, et al., 2003 \\
\hline T-Sapphire & 399 & 511 & 44.0 & 0.60 & Monomer* & 79 & Zapata-Hommer, et al., 2003 \\
\hline \multicolumn{8}{|c|}{ Cyan Fluorescent Proteins } \\
\hline ECFP & 439 & 476 & 32.5 & 0.40 & Monomer* & 39 & Cubitt, et al., 1995 \\
\hline mCFP & 433 & 475 & 32.5 & 0.40 & Monomer & 39 & Zacharias, et al., 2002 \\
\hline Cerulean & 433 & 475 & 43.0 & 0.62 & Monomer* & 79 & Rizzo, et al., 2004 \\
\hline CyPet & 435 & 477 & 35.0 & 0.51 & Monomer* & 53 & Nguyen and Daugherty, 2005 \\
\hline AmCyan1 & 458 & 489 & 44.0 & 0.24 & Tetramer & 31 & Matz, et al., 1999 \\
\hline Midoriishi Cyan & 472 & 495 & 27.3 & 0.90 & Dimer & 73 & Karasawa, et al., 2004 \\
\hline \multicolumn{8}{|c|}{ Green Fluorescent Proteins } \\
\hline EGFP & 484 & 507 & 56.0 & 0.60 & Monomer* & 100 & Heim, et al., 1995 \\
\hline aceGFP & 480 & 505 & 50.0 & 0.55 & Monomer* & 82 & Gurskaya, et al., 2003 \\
\hline TurboGFP & 482 & 502 & 70.0 & 0.53 & Monomer* & 110 & Shagin, et al., 2004 \\
\hline Emerald & 487 & 509 & 57.5 & 0.68 & Monomer* & 116 & Cubitt, et al., 1999 \\
\hline Azami Green & 492 & 505 & 55.0 & 0.74 & Monomer & 121 & Karasawa, et al., 2003 \\
\hline ZsGreen & 493 & 505 & 43.0 & 0.91 & Tetramer & 117 & Matz, et al, 1999 \\
\hline \multicolumn{8}{|c|}{ Yellow Fluorescent Proteins } \\
\hline EYFP & 514 & 527 & 83.4 & 0.61 & Monomer* & 151 & Miyawaki, et al., 1999 \\
\hline Topaz & 514 & 527 & 94.5 & 0.60 & Monomer* & 169 & Tsien, 1998 \\
\hline Venus & 515 & 528 & 92.2 & 0.57 & Monomer* & 156 & Nagai, et al., 2002 \\
\hline mCitrine & 516 & 529 & 77.0 & 0.76 & Monomer & 174 & Griesbeck, et al., 2001 \\
\hline YPet & 517 & 530 & 104 & 0.77 & Monomer* & 238 & Nguyen and Daugherty, 2005 \\
\hline PhiYFP & 525 & 537 & 124 & 0.39 & Monomer* & 144 & Shagin, et al., 2004 \\
\hline ZsYellow1 & 529 & 539 & 20.2 & 0.42 & Tetramer & 25 & Matz, et al., 1999 \\
\hline mBanana & 540 & 553 & 6.00 & 0.70 & Monomer & 13 & Shaner, et al., 2004 \\
\hline \multicolumn{8}{|c|}{ Orange and Red Fluorescent Proteins } \\
\hline Kusabira Orange & 548 & 559 & 51.6 & 0.60 & Monomer & 92 & Karasawa, et al., 2004 \\
\hline mOrange & 548 & 562 & 71.0 & 0.69 & Monomer & 146 & Shaner, et al., 2004 \\
\hline dTomato & 554 & 581 & 69.0 & 0.69 & Dimer & 142 & Shaner, et al., 2004 \\
\hline dTomato-Tandem & 554 & 581 & 138 & 0.69 & Monomer & 283 & Shaner, et al., 2004 \\
\hline DsRed & 558 & 583 & 75.0 & 0.79 & Tetramer & 176 & Matz, et al., 1999 \\
\hline DsRed2 & 563 & 582 & 43.8 & 0.55 & Tetramer & 72 & Bevis and Glick, 2002 \\
\hline DsRed-Express (T1) & 555 & 584 & 38.0 & 0.51 & Tetramer & 58 & Bevis and Glick, 2002 \\
\hline DsRed-Monomer & 556 & 586 & 35.0 & 0.10 & Monomer & 10 & Clontech, 2005 \\
\hline tangerine & 568 & 585 & 38.0 & 0.30 & Monomer & 34 & Shaner, et al., 2004 \\
\hline mStrawberry & 574 & 596 & 90.0 & 0.29 & Monomer & 78 & Shaner, et al., 2004 \\
\hline AsRed2 & 576 & 592 & 56.2 & 0.05 & Tetramer & 8 & Matz, et al., 1999 \\
\hline mRFP1 & 584 & 607 & 50.0 & 0.25 & Monomer & 37 & Campbell, et al., 2002 \\
\hline JRed & 584 & 610 & 44.0 & 0.20 & Dimer & 26 & Shagin, et al., 2004 \\
\hline mCherry & 587 & 610 & 72.0 & 0.22 & Monomer & 47 & Shaner, et al., 2004 \\
\hline HcRed1 & 588 & 618 & 20.0 & 0.015 & Dimer & 1 & Gurskaya, et al., 2001 \\
\hline mRaspberry & 598 & 625 & 86.0 & 0.15 & Monomer & 38 & Wang, et al., 2004 \\
\hline HcRed-Tandem & 590 & 637 & 160 & 0.04 & Monomer & 19 & Fradkov, et al., 2002 \\
\hline mPlum & 590 & 649 & 41.0 & 0.10 & Monomer & 12 & Wang, et al., 2004 \\
\hline AQ143 & 595 & 655 & 90.0 & 0.04 & Tetramer & 11 & Shkrob, et al., 2005 \\
\hline
\end{tabular}


Table 1. A compilation of properties displayed by several of the most popular and useful fluorescent protein variants. Along with the common name and/or acronym for each fluorescent protein, the peak excitation (Ex) and emission (Em) wavelengths, molar extinction coefficient (EC), quantum yield (QY), relative brightness, and in vivo structural associations are listed. The computed brightness values were derived from the product of the molar extinction coefficient and quantum yield, divided by the value for EGFP. This listing was created from scientific and commercial literature resources and is not intended to be comprehensive, but instead represents fluorescent protein derivatives that have received considerable attention in the literature and may prove valuable in research efforts. The excitation and emission peak values listed may vary in published reports due to the broad spectral profiles. In actual fluorescence microscopy investigations, spectral profiles and wavelength maxima are influenced by environmental effects, such as $\mathrm{pH}$, ionic concentration, and solvent polarity, as well as fluctuations in localized probe concentration. Therefore, the listed extinction coefficients and quantum yields may differ from those actually observed under experimental conditions.

The first Anthozoa-derived fluorescent protein to be extensively investigated was derived from the sea anemone Discosoma striata and originally referred to as drFP583, but is now commonly known as DsRed (Matz, et al., 1999). Once the protein has fully matured, the fluorescence emission spectrum of DsRed features a peak at $583 \mathrm{~nm}$ whereas the excitation spectrum has a major peak at $558 \mathrm{~nm}$ and a minor peak around $500 \mathrm{~nm}$. Several problems are associated with DsRed in practice. Maturation of DsRed fluorescence occurs slowly and proceeds through an intermediate chromophore stage where a majority of the fluorescence emission is seen in the green region (Baird, et al., 2000). Termed the "green state", this artifact has proven challenging for multiple labeling experiments in combination with other green fluorescent proteins because of the spectral overlap. In addition, DsRed is an obligate tetramer, an undesirable characteristic that interferes in fusion protein constructs, often leading to poor localization, and increases the tendency to form large protein aggregates in living cells. Although these side effects are not important when the probe is utilized simply as a reporter for gene expression, the utility of DsRed as an epitope tag is severely compromised (Rizzo and Piston, 2005). In contrast to the large Aequorea family of proteins that have been employed to successfully tag hundreds of fusion proteins, DsRed fusion proteins have proven far less successful and often exhibit toxic effects.

Several of the major problems with DsRed fluorescent protein have been overcome through sitedirected and random mutagenesis efforts. The second-generation version of DsRed, known as DsRed2, contains a series of silent nucleotide substitutions corresponding to human codon preferences and several internal sequence mutations that increase the maturation rate (Bevis and Glick, 2002). In addition, the elimination of basic amino acid residues (changed to acidic or neutral moieties) at the peptide amino terminus in DsRed2 reduces the tendency of the protein to form aggregates in fusion constructs (Yanushevich, et al., 2002). The DsRed2 protein still forms a tetramer in solution, but it is more compatible with green fluorescent proteins in multiple labeling experiments due to the increased maturation rate (Rizzo and Piston 2005a). Further reductions in maturation time have been realized with the third generation of DsRed mutants, which also display an increased brightness level in terms of peak cellular fluorescence. Red fluorescence emission from DsRed-Express (a commercial vector from Clontech) can be observed within an hour after expression, as compared to approximately six hours for DsRed2 and 11 hours for DsRed (Bevis and Glick, 2002; Rizzo and Piston, 2005a). The presence of a green state in DsRedExpress is not apparent, rendering this fluorescent protein the best choice of tetrameric DsRed variants for multiple labeling experiments. Because these probes remain obligate tetramers, however, they have largely been replaced with dimeric and monomeric versions and are now only of historical interest. A commercially available monomeric version of DsRed (Clontech) has recently been introduced, but the intrinsic brightness and photostability of this probe are reported to be poor (Shaner, et al., 2005).

Several additional red fluorescent proteins showing a considerable amount of promise have been isolated from the reef coral organisms. One of the first to be adapted for mammalian cell applications is HcRed1 (Gurskaya, et al., 2001), which was isolated from the anemone Heteractis crispa and is now commercially available (Clontech and Evrogen). HcRed1 was originally derived from a non-fluorescent chromoprotein that absorbs orange light through site-directed and random mutagenesis. A total of six amino acid substitutions were necessary to create a red fluorescent species that matured rapidly and efficiently at $37^{\circ} \mathrm{C}$ (absorption and emission at 588 and $618 \mathrm{~nm}$, respectively). However, similar to many other reef coral proteins, the resulting red fluorescent HcRed displays a tendency to form obligate tetramers 
when expressed in bacteria. Additional mutagenesis efforts resulted in a brighter dimeric variant (Gurskaya, et al., 2001), but a monomeric version of the protein has not yet been discovered. In order to generate a species of the protein that is useful in creating fusion products for localization studies, a tandem dimer expression vector of HcRed (two head-to-tail identical copies of the protein) has been constructed (Fradkov, et al., 2002). When fused to a gene product that itself forms biopolymers (such as actin or tubulin), the HcRed tandem dimer forms an intramolecular self-association complex (mimicking a monomeric tag) that apparently does not interfere with the biological activity of the resulting chimera. However, because the overall brightness and photostability of this twinned protein combination has not yet been improved, it remains a secondary choice for routine applications in live-cell microscopy.

\section{Filter Combination for Multicolor Imaging of Fluorescent Proteins}
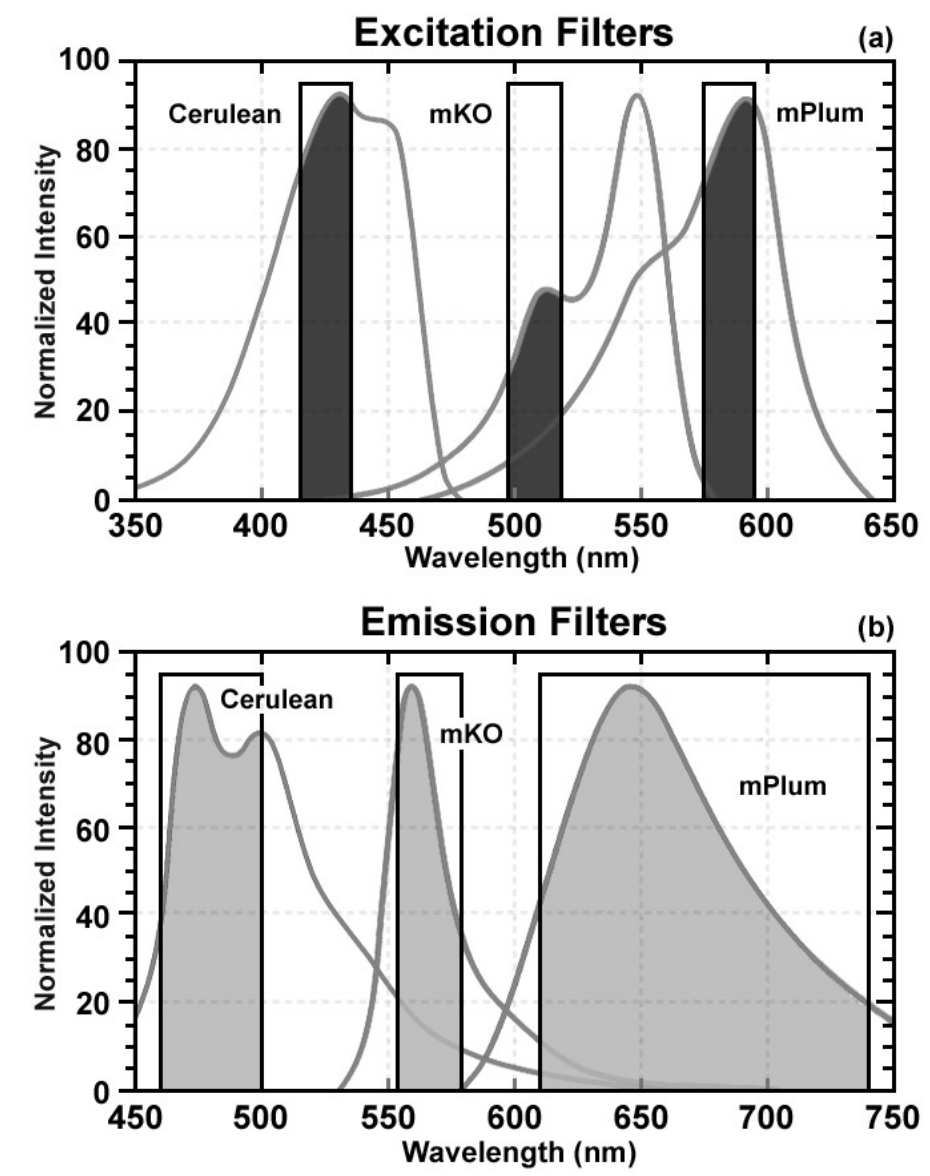

Figure 4. Optimized fluorescence filter combinations for multicolor imaging of three fluorescent proteins spanning the cyan through far-red wavelength regions. (a) Excitation filters optimized for Cerulean, mKO, and mPlum fluorescent proteins having center wavelengths of 425,508 , and $585 \mathrm{~nm}$, respectively. The bandwidth of all excitation filters is 20 $\mathrm{nm}$. (b) Emission filters optimized for the same probes having center wavelengths of 480, 564, and $675 \mathrm{~nm}$ with bandwidths of 40, 28, and $130 \mathrm{~nm}$, respectively. The mKO excitation filter is designed to work off-peak to minimize simultaneous excitation of mPlum. Bleed-through of Cerulean fluorescence emission into the mKO filter is reduced by the fact that $\mathrm{mKO}$ is four times brighter at equimolar concentrations and requires much shorter exposure intervals for image capture.

A far-red fluorescent protein, termed eqFP611, was isolated from the sea anemone Entacmaea quadricolor and displays one of the largest Stokes shifts and red-shifted fluorescence emission wavelength profiles (excitation and emission maxima at 559 and $611 \mathrm{~nm}$, respectively) of any naturally occurring 
Anthozoan fluorescent protein (Wiedenmann, et al., 2002). The quantum yield and extinction coefficient of eqFP611 combine to yield a probe approximately as bright as EGFP. During the in vivo fluorophore maturation process, which occurs in about 12 hours, the protein passes through a green intermediate state. After maturation, however, only a small fraction of this green species (less than 1 percent) can be detected. In contrast to other Anthozoan fluorescent proteins, eqFP611 has a reduced tendency to oligomerize at lower concentrations as evidenced through electrophoresis and single-molecule experiments (Schenk, et al., 2004), although at high concentrations the protein does form tetramers. Site-directed mutagenesis efforts have yielded functional dimeric variants of eqFP611 (Wiedenmann, et al., 2005), and continued efforts may finally lead to a monomeric far-red fluorescent protein from this species.

Two additional reef coral red fluorescent proteins, AsRed2 and JRed, are commercially available (Clontech and Evrogen), but these probes form tetrameric and dimeric complexes, respectively, and are less useful than the monomeric proteins described below. AsRed2 was originally isolated as a chromoprotein from Anemonia sulcata (Matz, et al., 1999) and modified through mutagenesis to yield a protein having an absorption maximum at $576 \mathrm{~nm}$ and an emission peak at $595 \mathrm{~nm}$ (Lukyanov, et al., 2000) with a very modest quantum yield (0.05). Although the protein has been optimized with human codons for expression in mammalian cell lines, it exhibits only about $10 \%$ the brightness level of EGFP and the photostability has not been reported. The dimeric protein, JRed, was derived through extensive mutagenesis of a jellyfish chromoprotein (Shagin, et al., 2004) to produce a novel red fluorescent marker with peak absorption and emission wavelengths of 584 and $610 \mathrm{~nm}$, respectively. The probe has been demonstrated to produce useful fusion tags, but is unsuitable for expression in prokaryotes due to folding problems. JRed is about $25 \%$ as bright as EGFP and exhibits limited photostability when illuminated in the 560 to $580 \mathrm{~nm}$ region, but can be successfully employed for long-term imaging experiments when excited with a 543-nm laser.

The construction of true monomeric DsRed variants, as well as monomers from proteins in other Anthozoa species, has proven to be a difficult task (Rizzo and Piston, 2005a). A total of 33 amino acid alterations to the DsRed sequence were required for the creation of the first-generation monomeric red fluorescent protein (termed RFP1; Campbell, et al., 2002). However, this derivative exhibits significantly reduced fluorescence emission compared to the native protein and photobleaches very quickly, rendering it much less useful than analogous monomeric green and yellow fluorescent proteins. Extensive mutagenesis research efforts (Shaner, et al., 2004), including novel techniques such as iterative somatic hypermutation (Wang, et al., 2004), have successfully been applied in the search for yellow, orange, red, and far-red fluorescent protein variants that further reduce the tendency of these potentially efficacious biological probes to self-associate while simultaneously pushing emission maxima towards longer wavelengths. The result has been improved monomeric fluorescent proteins that feature increased extinction coefficients, quantum yields, and photostability, although no single variant has yet been optimized by all criteria. In addition, the expression problems with obligate tetrameric red fluorescent proteins are being overcome by the efforts to generate true monomeric variants, which have yielded derivatives that are more compatible with biological function.

Perhaps the most spectacular development on this front has been the introduction of a new harvest of fluorescent proteins (Patterson, 2004) derived from monomeric red fluorescent protein (mRFP1) through directed mutagenesis targeting the Q66 and Y67 chromophore residues (Shaner, et al., 2004), which have been demonstrated to play key roles in determining the spectral characteristics in Aequorea proteins. The resulting cadre of monomeric fluorescent proteins exhibit maxima at wavelengths ranging from 560 to 610 $\mathrm{nm}$ and have been named in honor of common fruits that bear colors similar to their respective fluorescence emission spectral profiles. Among the potentially efficacious members in the "fruit" series of fluorescent proteins are mStrawberry, mCherry, and tdTomato (a tandem dimer), all of which have fluorescence emission profiles in the orange and red regions of the spectrum. Of these probes, tdTomato, which has an orange-red emission maximum at $581 \mathrm{~nm}$, is the brightest and most photostable under widefield illumination (Shaner, et al., 2005), although the dimer is twice the molecular weight of the monomers. The red proteins, mCherry and mStrawberry (emission peaks at 610 and $596 \mathrm{~nm}$, respectively), have brightness levels of approximately $50 \%$ and $75 \%$ of EGFP, but mCherry is far more photostable than mStrawberry and is the best probe choice to replace mRFP1 for long-term imaging experiments. These new proteins essentially fill the gap between the most red-shifted jellyfish fluorescent proteins (such as Venus) and the multitude of oligomeric coral reef red fluorescent proteins that have been reported and are commercially 
available. Although several of these new fluorescent monomeric proteins lack the brightness and photostability necessary for many imaging experiments (Shaner, et al., 2005; Tsien, 2005), their existence is encouraging as it suggests the eventuality of bright, stable, monomeric fluorescent proteins across the entire visible spectrum.

\section{Far-Red Fluorescent Proteins}

Further extension of the fruit protein spectral class through iterative somatic hypermutation (Wang, et al., 2004) has yielded two new fluorescent proteins with emission wavelength maxima of 625 and $649 \mathrm{~nm}$, representing the first true far-red (ranging from 630 to $700 \mathrm{~nm}$ ) genetically engineered probes. The most potentially useful probe in this pair was named mPlum, which has a rather limited brightness value (10\% of EGFP), but excellent photostability. This monomeric probe should be useful in combination with fluorescent proteins emitting in the cyan, green, yellow, and orange regions for multicolor imaging experiments (see Figure 4) and as a biosensor FRET partner with green and yellow proteins, such as mEmerald (Förster distance, 4.4) and mCitrine (Förster distance, 5.0). Another far-red fluorescent protein, termed AQ143, has been derived from mutagenesis efforts on a chromoprotein isolated from the anemone Actinia equine (Shkrob, et al., 2005). The excitation and emission maxima of AQ143 are 595 and $655 \mathrm{~nm}$, respectively, and the brightness is comparable to mPlum. On the downside, the photostability of this protein has not been reported and it forms an obligate tetramer.

\section{Optical Highlighter Fluorescent Proteins}

Protein chromophores that can be activated to initiate fluorescence emission from a quiescent state (a process known as photoactivation), or are capable of being optically converted from one fluorescence emission bandwidth to another (photoconversion), represent perhaps the most promising approach to the in vivo investigation of protein lifetimes, transport, and turnover rates. Appropriately termed molecular or optical highlighters, photoactivated fluorescent proteins generally display little or no initial fluorescence under excitation at the imaging wavelength, but dramatically increase their fluorescence intensity after activation by irradiation at a different (usually lower) wavelength (for reviews see: Lippincott-Schwartz, et al., 2003; Lukyanov, et al., 2005; Miyawaki, 2004). Photoconversion optical highlighters, on the other hand, undergo a change in the fluorescence emission bandwidth profile upon optically-induced changes to the chromophore. These effects result in the direct and controlled highlighting of distinct molecular pools within the cell. Because only the limited population of photoactivated molecules exhibit noticeable fluorescence, their lifetime and behavior can be followed independently of other proteins that are newly synthesized. In a similar manner, fluorescent proteins that are capable of being optically modulated through photoconversion to reversibly alter emission intensity and/or color are also potentially very useful for examining dynamic processes in living cells.

The ideal optical highlighter proteins should be readily photoconvertible (through the process of fluorescence activation and/or emission wavelength shifts) to produce a high level of contrast, as well as being monomeric for optimum expression in the target system. These probes will be especially useful in experiments paralleling results obtained with photobleaching techniques, such as recovery (FRAP) and loss (FLIP) of photobleaching, because they have the advantage that measurements are not influenced by newly synthesized or non-converted proteins, which either remain invisible or continue to emit the original wavelengths (Lippincott-Schwartz, et al., 2003). Also, by repeated excitation in the region of interest, optical highlighters can be continuously photoconverted at a specific intracellular location. This technique is more efficient than FLIP because the translocation of activated proteins can be directly imaged. In addition, the time required for photoactivation (a few seconds) is often much less than the time required to completely photobleach a similar region. Investigations involving extremely rapid cellular processes will clearly benefit from such improvements in temporal resolution. Presented in Table 2 is a compilation of important data for optical highlighter fluorescent proteins that display significant potential in applications as in vivo probes targeting cellular structure and function. Included are the excitation (or absorption) and fluorescence emission maximum wavelengths for both the activated and non-activated species, as well as the molar extinction coefficients, quantum yields, molecular structure (monomer, dimer, tetramer, etc.), and relative brightness level compared to enhanced green fluorescent protein (EGFP). Also listed are 
references to the original literature sources and review articles. This table should serve as a convenient guide for comparing the properties of optical highlighters.

The first useful optical highlighter designed specifically for photoactivation studies is a variant of the jellyfish (Aequorea victoria) green fluorescent protein, termed PA-GFP (an acronym for Photo Activatable Green Fluorescent Protein). This photoactivatable version of GFP was developed by improving on the photoconversion efficiency of the natural wild-type protein chromophore from a predominately neutral form to a species that is anionic in character (Lippincott-Schwartz and Patterson, 2003; Patterson and Lippincott-Schwartz, 2002a, 2002b). Substitution of histidine for threonine at position 203 produced a variant with negligible absorbance in the region between 450 and $550 \mathrm{~nm}$, thus dramatically enhancing the contrast between the non-activated and activated species. After photoactivation with violet light, the absorption maximum at $504 \mathrm{~nm}$ in PA-GFP increases approximately 100 -fold. This event evokes very high contrast differences between the converted and unconverted pools of PA-GFP, and is useful for tracking the dynamics of molecular subpopulations within a cell. Note that after photoactivation, both PA-GFP and the wild-type GFP exhibit comparable levels of fluorescence when illuminated with a 488-nm laser. On the downside, intracellular targets expressing PA-GFP are not readily distinguishable before photoconversion without the use of a low-intensity violet illumination source (such as a 405-nanometer blue diode laser), making it difficult to identify the proper regions to be selectively targeted in photoactivation experiments using microscopes having only blue and green lasers.

\begin{tabular}{|c|c|c|c|c|c|c|c|}
\hline $\begin{array}{c}\text { Protein } \\
\text { (Acronym) }\end{array}$ & $\begin{array}{c}E x \\
(\mathbf{n m})\end{array}$ & $\begin{array}{c}\text { Em } \\
(\mathbf{n m})\end{array}$ & $\begin{array}{c}\text { EC } \\
\times 10^{-3}\end{array}$ & QY & $\begin{array}{c}\text { in vivo } \\
\text { Structure }\end{array}$ & $\begin{array}{c}\text { Relative } \\
\text { Brightness } \\
\text { (\% of EGFP) }\end{array}$ & References \\
\hline PA-GFP (G/NA) & 400 & 515 & 20.7 & 0.13 & Monomer & 8 & Patterson, et al., 2002 \\
\hline PA-GFP (G) & 504 & 517 & 17.4 & 0.79 & Monomer & 41 & Patterson, et al., 2002 \\
\hline PS-CFP (C) & 402 & 468 & 34.0 & 0.16 & Monomer & 16 & Chudakov, et al., 2004 \\
\hline PS-CFP (G) & 490 & 511 & 27.0 & 0.19 & Monomer & 15 & Chudakov, et al., 2004 \\
\hline PS-CFP2 (C) & 400 & 468 & 43.0 & 0.20 & Monomer & 26 & Chudakov, et al., 2004 \\
\hline PS-CFP2 (G) & 490 & 511 & 47.0 & 0.23 & Monomer & 32 & Chudakov, et al., 2004 \\
\hline PA-mRFP1 (R) & 578 & 605 & 10.0 & 0.08 & Monomer & 3 & Verkhusha, et al., 2005 \\
\hline Kaede (G) & 508 & 518 & 98.8 & 0.88 & Tetramer & 259 & Ando, et al., 2002 \\
\hline Kaede (R) & 572 & 580 & 60.4 & 0.33 & Tetramer & 59 & Ando, et al., 2002 \\
\hline Kikume (KikGR; G) & 507 & 517 & 53.7 & 0.70 & Tetramer & 112 & Tsutsui, et al., 2005 \\
\hline Kikume (KikGR; R) & 583 & 593 & 35.1 & 0.65 & Tetramer & 68 & Tsutsui, et al., 2005 \\
\hline mEosFP (G) & 505 & 516 & 67.2 & 0.64 & Monomer & 128 & Wiedenmann, et al., 2004 \\
\hline $\mathrm{mEosFP}(\mathrm{R})$ & 569 & 581 & 37.0 & 0.62 & Monomer & 68 & Wiedenmann, et al., 2004 \\
\hline Kindling (R) & 580 & 600 & 59.0 & 0.07 & Tetramer & 12 & Labas, et al., 2002 \\
\hline Dronpa (G) & 503 & 518 & 95.0 & 0.85 & Monomer & 240 & Ando, et al., 2004 \\
\hline
\end{tabular}

Table 2. A compilation of important data for fluorescent proteins that display significant potential in applications as in vivo probes targeting cellular structure and function. Included are the excitation (Ex) and fluorescence emission (Em) maximum wavelengths for both the activated and non-activated species, as well as the molar extinction coefficients (EC), quantum yields (QY), molecular structure (monomer, dimer, tetramer, etc.), and relative brightness level compared to enhanced EGFP. Also listed are references to the original literature sources.

Several new photoactivatable proteins have been produced using site-directed mutagenesis of monomeric red-shifted reef coral fluorescent proteins. In particular, the monomeric derivative of DsRed fluorescent protein, mRFP1, has been converted into probes that are photoactivated by either green or violet irradiation (Verkhusha and Sorkin, 2005). These have been collectively termed PA-mRFP1s, the brightest of which exhibits a 70-fold increase of fluorescence intensity upon activation by wavelengths between 380 and $400 \mathrm{~nm}$. The critical molecular determinant residues for producing photoactivatable proteins with coral 
reef variants appear to be the amino acids at positions 146, 161, and 197 (DsRed numbering scheme). These residues, which lay in close proximity to the chromophore, serve to stabilize the tyrosine residue at position 66 in either a cis (fluorescent) or trans (non-fluorescent) configuration to form a fluorescent protein (cis) or a chromoprotein (trans) that absorbs light but does not emit fluorescence. The monomeric red fluorescent protein optical highlighters have been demonstrated to be useful in some cases as a fusion tag in mammalian systems for investigation of intracellular dynamics. However, the relatively low level of fluorescence of PA-mRFP1s in the photoactivated form (3 percent of EGFP), along with a tendency to form non-specific aggregates when fused to naturally oligomeric proteins (for example, actin and tubulin), render these probes less useful than PA-GFP for live-cell investigations (Verkhusha and Sorkin, 2005). Improved monomeric yellow, orange, and red fluorescent proteins (such as mCherry, mStrawberry, and mPlum) hold the potential to produce efficient photoactivatable optical highlighters with emission wavelengths extending into the deep red portion of the visible light spectrum.

A novel photoconversion optical highlighter, termed photoswitchable cyan fluorescent protein (PS-CFP), derived from the Aequorea coerulescens green fluorescent protein variant, aceGFP, has been observed to transition from cyan $(468 \mathrm{~nm})$ to green fluorescence $(511 \mathrm{~nm})$ upon illumination at $405 \mathrm{~nm}$. The PS-CFP highlighter was generated by site-directed mutagenesis of aceGFP (Chudakov, et al., 2004). Expressed as a monomer in vivo, this probe should be useful in photobleaching, photoconversion, and photoactivation investigations because it can potentially be fused to a wide variety of proteins without altering their behavior. Another advantage of PS-CFP is the significant level of cyan fluorescence that is present before photoconversion, a factor that allows investigators to track and selectively illuminate specific intracellular regions or entire cells for study. However, the fluorescence emission intensity from PS-CFP is approximately 2.5-fold dimmer than PA-GFP, and the probe is inferior to other highlighters in terms of photoconversion efficiency (the 40-nanometer wavelength shift in fluorescence emission upon photoconversion is less than that observed with similar optical highlighters). A high-contrast, brighter variant of PS-CFP, designated PS-CFP2, is commercially available (Evrogen) as a cloning vector.

Several interesting and potentially useful optical highlighters have been developed in fluorescent proteins cloned from reef coral and sea anemone species. One of the first and most important examples, a tetrameric fluorescent protein isolated from the stony Open Brain coral, Trachyphyllia geoffroyi, has been found to photoconvert from green to red fluorescence emission in the presence of ultraviolet light (Ando, et al., 2002). It is interesting to note that the discovery of this highlighter occurred when researchers accidentally left a test tube containing the protein on a laboratory bench near a window, and then astutely observed the shift from green to red. The unusual color transition prompted investigators to name the protein Kaede, after the leaves of the Japanese maple tree, which turn from green to red in the fall months. Irradiation of the commercially available (MBL) Kaede optical highlighter with light between 380 and 400 $\mathrm{nm}$ results in a rapid spectral shift from principal maxima at $508 \mathrm{~nm}$ (absorption) and $518 \mathrm{~nm}$ (emission) to longer wavelength peaks at 572 and $582 \mathrm{~nm}$, respectively. Upon photoconversion, Kaede exhibits a dramatic increase in the red-to-green fluorescence ratio (approximately 2000-fold, considering both the decrease in green and the increase in red emission). The conversion is stable and irreversible under aerobic conditions. Neither exposure to dark for extended periods nor strong illumination at $570 \mathrm{~nm}$ is able to restore green fluorescence to the chromophore. The red fluorescent state of the Kaede chromophore is comparable to the green in terms of brightness and stability, and because the unconverted protein emits very little fluorescence above $550 \mathrm{~nm}$, the appearance of strong red signal provides excellent contrast.

Similar proteins capable of being photoconverted by violet and ultraviolet illumination have been discovered in the Great Star coral (mcavRFP; derived from Montastraea cavernosa; Shagin, et al., 2004), soft corals (DendFP; derived from members of the genus Dendronephthya; Pakhomov, et al., 2004), and the mushroom coral (rfloRFP; derived from Ricordea florida; Shagin, et al., 2004). All of these highlighters (including Kaede, Eos, and KikGR, discussed below) contain a chromophore derived from the tripeptide His-Tyr-Gly that initially emits green fluorescence until driven into a red state by light rather than chemical oxidation, as is the case with many red fluorescent proteins derived from reef corals. These amino acids form the imidazolinone chromophore (similar to the Aequorea proteins) that is transformed from green to red fluorescence emission under illumination at shorter visible and longer ultraviolet wavelengths. Irradiation induces cleavage between the amide nitrogen and alpha carbon atoms in the histidine residue with subsequent formation of a highly conjugated dual imidazole ring system, a process 
requiring catalysis by the intact protein and resulting in the dramatic shift of fluorescence emission to red wavelengths (Mizuno, et al., 2003; Nienhaus, et al., 2005). The unconventional chemistry involved in this chromophore transition should serve fluorescent protein engineers with an excellent foundation upon which to develop more advanced highlighters.

\section{Optical Highlighter Fluorescent Proteins}
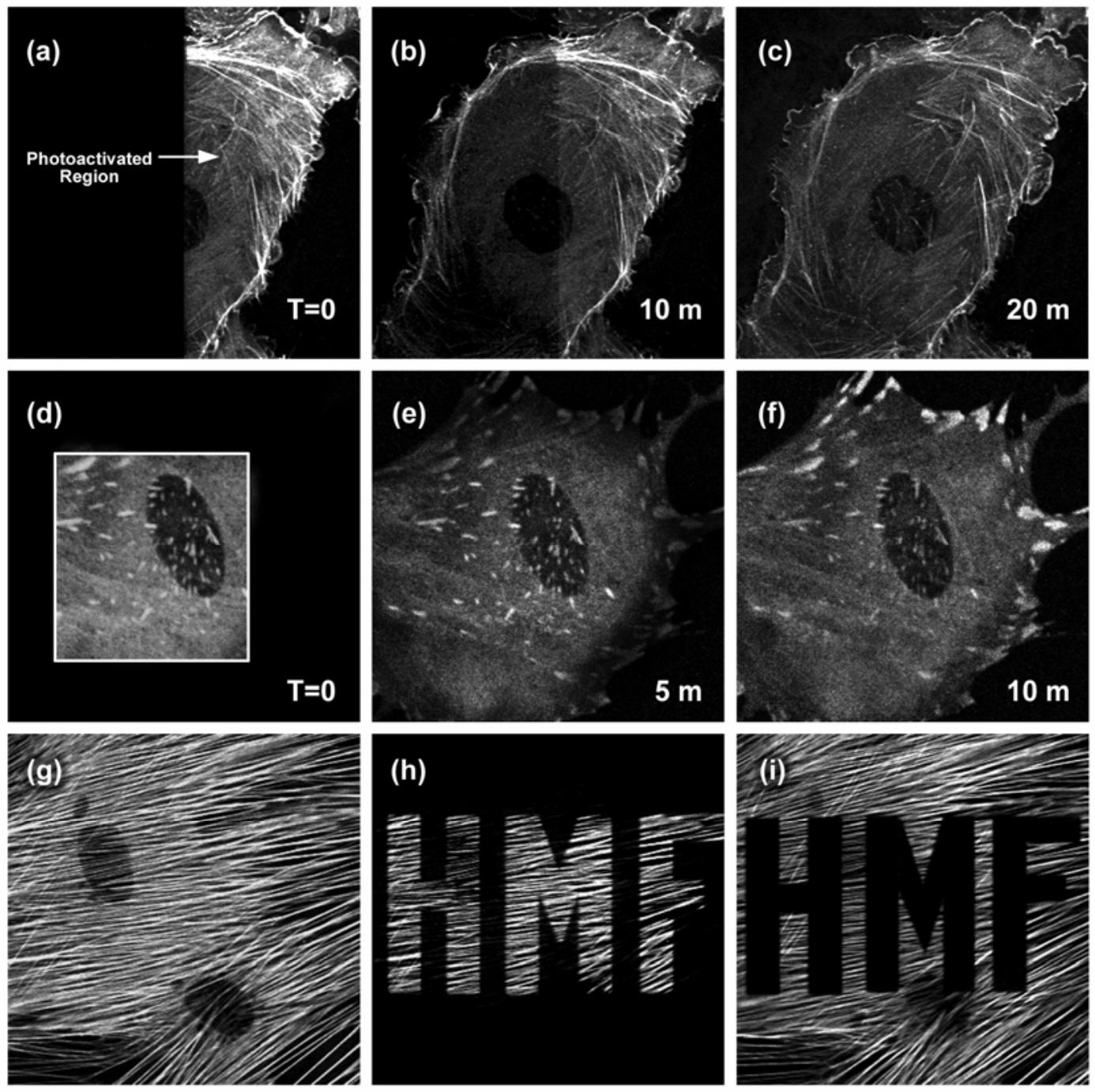

Figure 4. Photoactivation, photoconversion, and photoswitching with optical highlighter fluorescent proteins. (a) - (c) PA-GFP fused to human $\beta$-actin expressed in an epithelial cell is illuminated at high intensity with a 405-nm diode laser in the right-hand portion of (a) to photoactivate the protein, which is slowly distributed across the filamentous network (b) and (c) over a period of 20 minutes. (d) - (f) A fusion tag containing EosFP and vinculin is localized to the focal adhesion regions in a fibroblast cell. After photoconversion of fluorescence from green to red in a selected region (d; only the red channel is illustrated), the converted highlighter diffuses through the cytoplasmic pool slowly making its way to the focal adhesion areas over a period of 10 minutes (e) and (f). (g) - (i) Photoswitching of Dronpa fused to human $\beta$-actin in a fibroblast. (g) Image of the actin cytoskeletal network with a 488-nm laser. (h) An AOTF was used to spell the letters "HMF" and the labeled molecules within the letters were photoswitched to the "on" state using a 405-nm diode laser after photoswitching molecules outside to the "off” state with a 488-nm laser. (i) Molecules inside the letters were photobleached with the 405-nm laser while imaging exterior molecules with the 488-nm laser. 
The stony coral Favia favus has yielded a promising tetrameric derivative that exhibits efficient photoconversion from green to red fluorescence emission wavelengths (similar to Kaede) upon irradiation with near-ultraviolet or violet light (Tsutsui, et al., 2005). Genetic engineering efforts based on structural analysis of this protein produced a variant, termed KikGR (named after Kikume-ishi, the Japanese term for Favia), which is several-fold brighter than Kaede in both the green and red states when expressed in mammalian cells. Commercially available (MBL) under the name Kikume Green-Red, the highlighter has been demonstrated to be successfully photoconverted using multiphoton excitation at $760 \mathrm{~nm}$, which can be used for specific labeling of cells with high spatial resolution in thick tissues. Furthermore, the Kikume highlighter features a wider separation of green and red emission maxima (75 nm vs. $54 \mathrm{~nm}$ ) than Kaede.

Another tetrameric stony coral (Lobophyllia hemprichii) fluorescent protein similar to Kaede, termed EosFP (named after the goddess of dawn in Greek mythology), emits bright green fluorescence at $516 \mathrm{~nm}$ that shifts to orange-red $(581 \mathrm{~nm})$ when illuminated with light at wavelengths in the nearultraviolet region of 390 to $405 \mathrm{~nm}$ (Wiedenmann, et al., 2004). Two single point mutations have been employed to split the wild-type tetramer into dimeric subunits, each of which is capable of being incorporated into functional fusion constructs with a variety of proteins that retain normal biological activity. By introducing a combination of both single point mutations into the wild-type EosFP tetramer, a true monomeric protein has been created that is referred to as mEosFP. Similar to the dimer subunits, the monomer is able to be incorporated into functional biological chimeras to serve as a marker in live-cell imaging (Ivanchenko, et al., 2005), although the monomer fusions are only efficiently expressed at temperatures below $30^{\circ} \mathrm{C}$ (limiting their applications in mammalian systems). Fluorescence spectroscopy of Eos indicates a $\mathrm{pH}$ dependence on photoconversion, suggesting that the protonated form of the chromophore is necessary for activation, which is consistent with an excited-state proton transfer mechanism.

The unique green-to-red optical highlighters discovered thus far in reef coral proteins certainly warrant an aggressive effort focused on solving the problems associated with aggregation, as well as finetuning photoactivation requirements and imaging spectral profiles. By engineering a variant that matures rapidly at $37^{\circ} \mathrm{C}$, the monomeric Eos protein, which features optical properties very similar to Kaede and KikGR, should be far more useful as an epitope tag due to the dramatically reduced tendency to form intermolecular associations between fusion proteins in vivo. In the future, by engineering optical highlighter proteins that shift photoconversion illumination wavelengths to the blue and green spectral regions, which are significantly less toxic to living cells, coupled with shifts of fluorescence emission to the yellow through far-red wavelength bands, the potential applications for Kaede, KikGR, and Eos derivative probes can be greatly expanded.

A useful non-Aequorea optical highlighter, the Kindling fluorescent protein (KFP1) has been developed from a non-fluorescent chromoprotein isolated in Anemonia sulcata (Chudakov, et al., 2003; Labas, et al., 2002), and is now commercially available (Evrogen). Kindling fluorescent protein does not exhibit emission until illuminated with green or yellow light in the region between 525 and $580 \mathrm{~nm}$. Lowintensity light results in transient red fluorescence (kindling) with excitation and emission maxima at 580 and $600 \mathrm{~nm}$, respectively, which slowly decays upon cessation of illumination as the protein relaxes back to its initial non-fluorescent state (exhibiting a half-life of approximately 50 to 60 seconds). Irradiation with intense blue light quenches the kindled fluorescence immediately and completely, allowing tight control over fluorescent labeling. Note that both kindling with low-intensity green light and quenching of fluorescence by blue light are reversible processes for the wild-type protein. In contrast, high-intensity illumination or continued irradiation at moderate levels results in irreversible kindling with a fluorescence intensity approximately 30 -fold greater than that of the non-activated protein. Irreversibly kindled molecules do not lose their fluorescence and are not quenched by illumination with blue light. This feature allows for stable long-term highlighting of cells, tissues, and organelles similar to PA-GFP and other highlighter proteins. The major drawback of kindling protein is its tendency to aggregate into tetramers, which seriously affects the potential for use as a protein fusion tag without some degree of disturbance to normal biological processes. However, the kindling protein is an excellent candidate for bulk photolabeling and tracking of individual organelles and cells within a large population. 
A new generation of specialized optical highlighters with reversible on-off switching capabilities was heralded by the introduction of Dronpa, a monomeric fluorescent protein derived from Pectiniidae, a family of stony reef corals featuring several members that naturally emit faint fluorescence upon irradiation with ultraviolet light (Ando, et al., 2004). Named after a fusion of the ninja term for vanishing (dron) and photoactivation (pa), Dronpa exhibits unusual photochromic behavior due to its ability to toggle fluorescence on and off by illumination with two different excitation wavelengths. Dronpa was engineered using both directed and random mutagenesis to generate a monomeric version of the wild-type oligomeric fluorescent protein having a major absorption maximum at $503 \mathrm{~nm}$ and a minor peak at $390 \mathrm{~nm}$ at neutral $\mathrm{pH}$. The absorption peak at $503 \mathrm{~nm}$ is due to the deprotonated species of the protein, while the smaller peak at $390 \mathrm{~nm}$ arises from the protonated form. When irradiated at $488 \mathrm{~nm}$, the fluorescence emission spectral profile of the deprotonated species has a maximum at $518 \mathrm{~nm}$ with a relatively high quantum yield of 0.85 . In contrast, the protonated from of the protein is almost non-fluorescent. Photoswitching of Dronpa occurs by interconversion between the deprotonated and protonated forms of the optical highlighter (Habuchi, et al., 2005). Upon intense irradiation at $488 \mathrm{~nm}$, the protein population is very efficiently driven to the protonated species with a commitment decrease in fluorescence to produce a dim (off) state in which the 390-nm absorption peak predominates. The dim state is readily converted back to the original fluorescent (on) deprotonated state with minimal illumination at $405 \mathrm{~nm}$.

Although photochromism (the ability to switch fluorescence on and off) has been observed in the wild-type and several yellow fluorescent protein variants of Aequorea victoria GFP at the single molecule level (Cinelli, et al., 2001; McAnaney, et al., 2005; Tinnefeld and Sauer, 2005), none have demonstrated this phenomenon when measured in bulk. In these studies, during several minutes of illumination at 488 $\mathrm{nm}$, the molecules produced fluorescence for several seconds, followed by an equally short time interval without emission, followed again by resumption of emission. Termed blinking behavior (Dickson, et al., 1997), this on-and-off switching sequence can be repeated a number of times before each green fluorescent protein molecule ultimately reaches a final long-lived non-fluorescent state upon the emission of approximately one million photons. The bright state can be recovered by irradiation with violet light (405 $\mathrm{nm}$ ) for a period of five minutes before re-initiating the sequence by illumination with a 488-nm laser. In this manner, many native green fluorescent protein derivatives display an optically induced switching effect in which each molecule is individually addressable. However, the ability of large Dronpa-tagged molecular ensembles (including organelles and entire cells) to collectively act as an information storage medium with the ability to write, erase, and read information nondestructively, and with high efficiency, is truly unique in the field of fluorescent proteins.

\section{The Future of Fluorescent Proteins}

The current thrust of fluorescent protein development is centered on fine-tuning the current palette of blue to yellow fluorescent proteins derived from the Aequorea victoria jellyfish, while simultaneously developing monomeric fluorescent proteins emitting in the orange to far-red regions of the visible light spectrum. Progress toward these goals has been substantial, and it is not inconceivable that near-infrared emitting fluorescent proteins loom on the horizon. The latest efforts in jellyfish variants have resulted in new and improved monomeric probes for the cyan, green, and yellow region, while the search for a bright monomeric and fast-maturing red fluorescent protein has yielded a host of excellent candidates spanning the longer wavelengths. Continuing protein engineering of the existing fluorescent proteins coupled with new technologies, such as the application of unnatural amino acids and circular permutation, should further expand the color palette.

The complex interplay of biological roles for an ever-increasing number of fluorescent proteins derived from a wide variety of marine species is only beginning to be understood. Light-induced changes to the autocatalytic chromophores, including photoactivation and photoconversion, may serve as a highly evolved photo-protection mechanism to assist these organisms in the useful dissipation of high-energy sunlight, especially the damaging shorter wavelengths, via the absorption and subsequent fluorescence reemission of longer and safer wavelengths. In many cases, these remarkable fluorescent proteins display very high photostability and dynamic photo-induced transformation properties, including spectral fine-

tuning of donor-acceptor pairs (and even cascades) for resonance energy transfer. The large number of spectral variants already discovered, featuring emission profiles covering the entire visible spectrum, 
suggests that the diverse optical and biochemical properties of these proteins will generate a host of new candidates as probes for biological investigations and ensure the continued development of unique genetically engineered fluorescent proteins.

As the development of optical highlighters continues, fluorescent proteins useful for optical marking should evolve towards brighter, monomeric derivatives with high contrast that can be easily photoconverted and display a wide spectrum of emission colors. For example, proteins capable of reversible photoactivation, red-to-green photoconversion, improved expression at elevated temperatures, and derivatives emitting in the far-red or near-infrared regions of the spectrum would be especially useful. Coupled with these advances, microscopes equipped to smoothly transition between illumination modes for fluorescence observation and regional marking will become commonplace in most cell biology laboratories. The combination of photoactivation and photoconversion with advanced fluorescence techniques, such as resonance energy transfer and multiphoton excitation, will enable the study of protein dynamics with greater precision and spatial resolution. Ultimately, these innovations have the potential to achieve significant advancements in the understanding of spatial and temporal dynamics in signal transduction systems.

\section{REFERENCES}

Alkaabi, K. M., Yafea, A., and Ashraf, S. S. 2005. Effect of pH on thermal- and chemical-induced denaturation of GFP, Applied Biochemistry and Biotechnology, 126, 149-156.

Ando, R., Hama, H., Yamamoto-Hino, M., Mizuno, H., and Miyawaki, A. 2002. An optical marker based on the UV-induced green-to-red-photoconversion of a fluorescent protein, Proceedings of the National Academy of Sciences, USA, 99, 12651-12656.

Ando, R., Mizuno, H., and Miyawaki, A. 2004. Regulated fast nucleocytoplasmic shuttling observed by reversible protein highlighting, Science, 306, 1370-1373.

Baird, G. S., Zacharias, D. A., and Tsien, R. Y. 2000. Biochemistry, mutagenesis, and oligomerization of DsRed, a red fluorescent protein from coral, Proceedings of the National Academy of Sciences, USA, 97, 11984-11989.

Beddoe, T., Ling, M., Dove, S., Hoegh-Guldberg, O., Devenish, R. J., Prescott, M., and Rossjohn, J. 2003. The production, purification and crystallization of a pocilloporin pigment from a reef-forming coral, Acta Crystallographica D: Biological Crystallography, 59, 597-599.

Bevis, B. J. and Glick, B. S. 2002. Rapidly maturing variants of the Discosoma red fluorescent protein (DsRed), Nature Biotechnology, 20, 83-87.

Boeck, G. 2001. Current status of flow cytometry in cell and molecular biology, International Review of Cytology, 204, 239-298.

Bulina, M. E., Chudakov, D. M., Britanova, O. V., Yanushevich, Y. G., Staroverov, D. B., Chepurnykh, T. V., Merzlyak, E. M., Shkrob, M. A., Lukyanov, S., and Lukyanov, K. A. 2006. A genetically encoded photosensitizer, Nature Biotechnology, 24, 95-99.

Bulina, M. E., Verkhusha, V. V., Staroverov, D. B., Chudakov, D. M., and Lukyanov, K. A. 2003. Heterooligomeric tagging diminishes non-specific aggregation of target proteins fused with Anthozoa fluorescent proteins, Biochemical Journal, 371, 109-114.

Campbell, R. E., Tour, O., Palmer, A. E., Steinbach, P. A., Baird, G. S., Zacharias, D. A., and Tsien, R. Y. 2002. A monomeric red fluorescent protein, Proceedings of the National Academy of Sciences, USA, 99, 7877-7882.

Chalfie, M., Tu, Y., Euskirchen, G., Ward, W. W., and Prasher, D. C. 1994. Green fluorescent protein as a marker for gene expression, Science, 263, 802-805.

Chattoraj, M., King, B. A., Bublitz, G. U., and Boxer, S. G. 1996. Ultra-fast excited state dynamics in green fluorescent protein: Multiple states and proton transfer, Proceedings of the National Academy of Sciences, USA, 93, 8362-8367.

Chudakov, D. M., Belousov, V. V., Zaraisky, A. G., Novoselov, V. V., Staroverov, D. B., Zorov, D. B., Lukyanov, S., and Lukyanov, K. A. 2003. Kindling fluorescent proteins for precise in vivo photolabeling, Nature Biotechnology, 21, 191-194.

Chudakov, D. M., Lukyanov, S., and Lukyanov, K. A. 2005. Fluorescent proteins as a toolkit for in vivo imaging, Trends in Biotechnology, 23, 605-613. 
Chudakov, D. M., Verkhusha, V. V., Staroverov, D. B., Souslova, E. A., Lukyanov, S., and Lukyanov, K. A. 2004. Photoswitchable cyan fluorescent protein for protein tracking, Nature Biotechnology, 22, 1435-1439.

Cinelli, R. A. G., Pellegrini, V., Ferrari, A., Faraci, P., Nifosi, R., Tyagi, M., Giacca, M., and Beltram, F. 2001. Green fluorescent proteins as optically controllable elements in bioelectronics, Applied Physics Letters, 79, 3353-3355.

Cody, C. W., Prasher, D. C., Westler, W. M., Prendergast, F. G., and Ward, W. W. 1993. Chemical structure of the hexapeptide chromophore of the Aequorea green-fluorescent protein, Biochemistry, 32, 1212-1218.

Cormier, M. J. and Eckroade, C. B. 1962. Studies on the bioluminescence of Renilla reniformis. III. Some biochemical comparisons to other Renilla species and determinations of the spectral energy distributions, Biochimica et Biophysica Acta, 64, 340-344.

Cornea, A. and Conn, P. M. 2002. Measurement of changes in fluorescence resonance energy transfer between gonadotropin-releasing hormone receptors in response to agonists, Methods, 27, 333-339.

Cubitt, A. B., Heim, R., Adams, S. R., Boyd, A. E., Gross, L. A., and Tsien, R. Y. 1995. Understanding, improving and using green fluorescent proteins, Trends in Biochemical Sciences, 20, 448-455.

Cubitt, A. H., Wollenweber, L. A., and Hein, R. 1999. Understanding structure-function relationships in the Aequorea victoria green fluorescent protein, Methods in Cell Biology, 58, 19-30.

Davenport, D. and Nichol, J. A. C. 1955. Luminescence in hydromedusae, Proceedings of the Royal Society of London, Series B, 144, 399-411.

Day, R. N. 2005. Imaging protein behavior inside the living cell, Molecular and Cellular Endocrinology, 230, 1-6.

Delagrave, S., Hawtin, R., Silva, C., Yang, M., and Youvan, D. 1995. Red-shifted excitation mutants of the green fluorescent protein, Nature Biotechnology, 13, 151-154.

Dickson, R. M., Cubitt, A. B., Tsien, R. Y., and Moerner, W. E. 1997. On/off blinking and switching behavior of single molecules of green fluorescent protein, Nature, 388, 355-358.

Dixit, R. and Cyr, R. 2003. Cell damage and reactive oxygen species production induced by fluorescence microscopy: Effect on mitosis and guidelines for non-invasive fluorescence microscopy, The Plant Journal, 36, 280-290.

Dixit, R., Cyr, R., and Gilroy, S. 2006. Using intrinsically fluorescent proteins for plant cell imaging, The Plant Journal, 45, 599-615.

Durand, R. E. and Olive, P. L. 1982. Cytotoxicity, mutagenicity, and DNA damage by Hoechst 33342, Journal of Histochemistry and Cytochemistry, 30, 111-116.

Edwards, A. M., Silva, E., Jofre, B., Becker, M. I., and De Ioannes, A. E. 1994. Visible light effects on tumoral cells in a culture medium enriched with tryptophan and riboflavin, Journal of Photochemistry and Photobiology B, 24, 179-186.

Fradkov, A. F., Verkhusha, V. V., Staroverov, D. B., Bulina, M. E., Yanushevich, Y. G., Martynov, V. I., Lukyanov, S., and Lukyanov, K. A. 2002. Far-red fluorescent tag for protein labeling, Biochemical Journal, 368, 17-21.

Greenbaum, L., Rothmann, C., Lavie, R., and Malik, Z. 2000. Green fluorescent protein photobleaching: A model for protein damage by endogenous and exogenous singlet oxygen, Biological Chemistry, 381, 1251-1258.

Griesbeck, O., Baird, G. S., Campbell, R. E., Zacharias, D. A., and Tsien, R. Y. 2001. Reducing the environmental sensitivity of yellow fluorescent protein: Mechanism and applications, Journal of Biological Chemistry, 276, 29188-29194.

Gross, L. A., Baird, G. S., Hoffman, R. C., Baldridge, K. K., and Tsien, R. Y. 2000. The structure of the chromophore within DsRed, a red fluorescent protein from coral, Proceedings of the National Academy of Sciences, USA, 97, 11990-11995.

Gurskaya, N. G., Fradkov, A. F., Pounkova, N. I., Staroverov, D. B., Bulina, M. E., Yanushevich, Y. G., Labas, Y. A., Lukyanov, S., and Lukyanov, K. A. 2003. A colorless green fluorescent protein homologue from the non-fluorescent hydromedusa Aequorea coerulescens and its fluorescent mutants, Biochemical Journal, 373, 403-408.

Gurskaya, N. G., Fradkov, A. F., Terskikh, A., Matz, M. V., Labas, Y. A., Martynov, V. I., Yanushevich, Y. G., Lukyanov, K. A., and Lukyanov, S. A. 2001. GFP-like chromoproteins as a source of far-red fluorescent proteins, Federation of the European Biochemical Societies (FEBS) Letters, 507, 16-20. 
Gurskaya, N. G., Savitsky, A. P., Yanushevich, Y. G., Lukyanov, S. A., and Lukyanov, K. A. 2001. Color transitions in coral's fluorescent proteins by site-directed mutagenesis, BMC Biochemistry, 2, 6-12.

Habuchi, S., Ando, R., Dedecker, P., Verheijen, W., Mizuno, H., Miyawaki, A., and Hofkens, J. 2005. Reversible single-molecule photoswitching in the GFP-like fluorescent protein Dronpa, Proceedings of the National Academy of Sciences, USA, 102, 9511-9516.

Heim, R., Cubitt, A. B., and Tsien, R. Y. 1995. Improved green fluorescence, Nature, 373, 663-664.

Heim, R., Prasher, D. C., and Tsien, R. Y. 1994. Wavelength mutations and post-translational autoxidation of green fluorescent protein, Proceedings of the National Academy of Sciences, USA, 91, 12501-12504.

Heim, R. and Tsien, R. Y. 1996. Engineering green fluorescent protein for improved brightness, longer wavelengths and fluorescence resonance energy transfer, Current Biology, 6, 178-182.

Ip., D. T. M., Chan, S. H., Allen, M. D., Bycroft, M., Wan, D. C. C., and Wong, K. B. 2004. Crystallization and preliminary crystallographic analysis of a novel orange fluorescent protein from the Cnidaria tube anemone Cerianthus sp., Acta Crystallographica D: Biological Crystallography, D60, 340-341.

Ivanchenko, S., Roecker, C., Oswald, F., Wiedenmann, J., and Nienhaus, G. U. 2005. Targeted green-red photoconversion of EosFP, a fluorescent marker protein, Journal of Biological Physics, 31, 249-259.

Jayaraman, S., Haggie, P., Wachter, R. M., Remington, S. J., and Verkman, A. S. 2000. Mechanism and cellular applications of green fluorescent protein-based halide sensor, Journal of Biological Chemistry, 275, 6047-6050.

Jung, G., Wiehler, J., and Zumbusch, A. 2005. The photophysics of green fluorescent protein: Influence of the key amino acids at positions 65, 203, and 222, Biophysical Journal, 88, 1932-1947.

Kajihara, D., Hohsaka, T., and Sisido, M. 2005. Synthesis and sequence optimization of GFP mutants containing aromatic non-natural amino acids at the Tyr66 position, Protein Engineering, Design and Selection, 18, 273-278.

Karasawa, S., Araki, T., Nagi, T., Mizuno, H., and Miyawaki, A. 2004. Cyan-emitting and orangeemitting fluorescent proteins as a donor/acceptor pair for fluorescence resonance energy transfer, Biochemical Journal, 381, 307-312.

Karasawa, S., Araki, T., Yamamoto-Hino, M., and Miyawaki, A. 2003. A green-emitting fluorescent protein from Galaxeidae coral and its monomeric version for use in fluorescent labeling, Journal of Biological Chemistry, 278, 34167-34171.

Khodjakov, A. and Rieder, C. L. 2006. Imaging the division process in living tissue culture cells, Methods, 38, 2-16.

Kuner, T. and Augustine, G. J. 2000. A genetically encoded ratiometric neurotechnique indicator for chloride: Capturing chloride transients in cultured hippocampal neurons, Neuron, 27, 447-459.

Labas, Y. A., Gurskaya, N. G., Yanushevich, Y. G., Fradkov, A. F., Lukyanov, K. A., Lukyanov, S. A., and Matz, M. V. 2002. Diversity and evolution of the green fluorescent protein family, Proceedings of the National Academy of Sciences, USA, 99, 4256-4261.

Lauf, U., Lopez, P., and Falk, M. M. 2001. Expression of fluorescently tagged connexins: A novel approach to rescue function of oligomeric DsRed-tagged proteins, Federation of the European Biochemical Societies (FEBS) Letters, 498, 11-15.

Lippincott-Schwartz, J., Altan-Bonnet, N., and Patterson, G. H. 2003. Photobleaching and photoactivation: Following protein dynamics in living cells, Nature Cell Biology, 5, S7-S14.

Lippincott-Schwartz, J. and Patterson, G. H. 2003. Development and use of fluorescent markers in living cells, Science, 300, 87-91.

Llopis, J., McCaffery, J. M., Miyawaki, A., Farquhar, M. G., and Tsien, R. Y. 1998. Measurement of cytosolic, mitochondria, and Golgi $\mathrm{pH}$ in single living cells with green fluorescent proteins, Proceedings of the National Academy of Sciences, USA, 95, 6803-6808.

Llopis, J., Westin, S., Ricote, M., Wang, J., Cho, C. Y., Kurokwa, R., Mullen, T. M., Rose, D. W., Rosenfeld, M. G., Tsien, R. Y., and Glass, C. K. 2000. Ligand-dependent interactions of coactivators steroid receptor coactivator-1 and peroxisome proliferator-activated receptor binding protein with nuclear hormone receptors can be imaged in live cells and are required for transcription, Proceedings of the National Academy of Sciences, USA, 97, 4363-4368.

Lucius, R., Mentlein, R., and Sievers, J. 1998. Riboflavin-mediated axonal degeneration of postnatal retinal ganglion cells in vitro is related to the formation of free radicals, Free Radicals in Biology and Medicine, 24, 798-808. 
Lukyanov, K. A., Chudakov, D. M., Lukyanov, S., and Verkhusha, V. V. 2005. Innovation: Photoactivatable fluorescent proteins, Nature Reviews Molecular Cell Biology, 6, 885-891.

Lukyanov, K. A., Fradkov, A. F., Gurskaya, N. G., Matz, M. V., Labas, Y. A., Savitsky, A. P., Markelov, M. L., Zaraisky, A. G., Zhao, X., Fang, Y., Tan, W., and Lukyanov, S. A. 2000. Natural animal coloration can be determined by a nonfluorescent green fluorescent protein homolog, Journal of Biological Chemistry, 275, 25879-25882.

Matz, M. V., Fradkov, A. F., Labas, Y. A., Savitsky, A. P., Zaraisky, A. G., Markelov, M. L., and Lukyanov, S. A. 1999. Fluorescent proteins from nonbioluminescent Anthozoa species, Nature Biotechnology, 17, 969-973.

McAnaney, T. B., Zeng, W., Doe, C. F. E., Bhanji, N., Wakelin, S., Pearson, D. S., Abbyad, P., Shi, X., Boxer, S. G., and Bagshaw, C. R. 2005. Protonation, photobleaching, and photoactivation of yellow fluorescent protein (YFP 10C): A unifying mechanism, Biochemistry, 44, 5510-5524.

Meyer, T. and Teruel, M. N. 2003. Fluorescence imaging of signaling networks, Trends in Cell Biology, 13, 101-106.

Minamikawa, T., Sriratana, A., Williams, D. A., Bowser, D. N., Hill, J. S., and Nagley, P. 1999. Chloromethyl-X-rosamine (MitoTracker Red) photosensitizes mitochondria and induces apoptosis in intact human cells, Journal of Cell Science, 112, 2419-2430.

Mitra, R. D., Silva, C. M., and Youvan, D. C. 1996. Fluorescence resonance energy transfer between blueemitting and red-shifted excitation derivatives of the green fluorescent protein, Gene, 173, 13-17.

Miyawaki, A. 2002. Green fluorescent protein-like proteins in reef Anthozoa animals, Cell Structure and Function, 27, 343-347.

Miyawaki, A. 2003. Fluorescence imaging of physiological activity in complex systems using GFP-based probes, Current Opinion in Neurobiology, 13, 591-596.

Miyawaki, A. 2004. Fluorescent proteins in a new light, Nature Biotechnology, 22, 1374-1376.

Miyawaki, A. 2005. Innovations in the imaging of brain functions using fluorescent proteins, Neuron, 48, 189-199.

Miyawaki, A., Griesbeck, O., Heim, R., and Tsien, R. Y. 1999. Dyanmic and quantitative calcium measurements using improved cameleons, Proceedings of the National Academy of Sciences, USA, 96, 2135-2140.

Miyawaki, A., Llopis, J., Heim, R., McCaffery, J. M., Adams, J. A., Ikura, M., and Tsien, R. Y. 1997. Fluorescent indicators for calcium based on green fluorescent proteins and calmodulin, Nature, 388, 882-887.

Miyawaki, A., Nagai, T., and Mizuno, H. 2005. Engineering fluorescent proteins, Advances in Biochemical Engineering and Biotechnology, 95, 1-15.

Mizuno, H., Mal, T. K., Tong, K. I., Ando, R., Furuta, T., Ikura, M., and Miyawaki, A. 2003. Photoinduced peptide cleavage in the green-to-red conversion of a fluorescent protein, Molecular Cell, 12, 1051-1058.

Mizuno, H., Swano, A., Eli, P., Hama, H., and Miyawaki, A. 2001. Red fluorescent protein from Discosoma as a fusion tag and a partner for fluorescence resonance energy transfer, Biochemistry, 40, 2502-2510.

Morin, J. G. and Hastings, J. W. 1971. Energy transfer in a bioluminescent system, Journal of Cellular Physiology, 77, 313-318.

Morin, J. G. and Hastings, J. W. 1977. Biochemistry of the bioluminescence of colonial hydroids and other coelenterates, Journal of Cellular Physiology, 77, 305-312.

Nagai, T., Ibata, K., Park, E. S., Kubota, M., Mikoshiba, K., and Miyawaki, A. 2002. A variant of yellow fluorescent protein with fast and efficient maturation for cell-biological applications, Nature Biotechnology, 20, 87-90.

Nagai, T. and Miyawaki, A. 2004. A high-throughput method for development of FRET-based indicators for proteolysis, Biochemical and Biophysical Research Communications, 319, $72-77$.

Nguyen, A. W. and Daugherty, P. S. 2005. Evolutionary optimization of fluorescent proteins for intracellular FRET, Nature Biotechnology, 23, 355-360.

Nienhaus, K., Nienhaus, G. U., Wiedenmann, J., and Nar, H. 2005. Structural basis for photo-induced protein cleavage and green to red conversion of fluorescent protein EosFP, Proceedings of the National Academy of Sciences, USA, 102, 9156-9159.

Ormö, M., Cubitt, A. B., Kallio, K., Gross, L. A., Tsien, R. Y., and Remington, S. J. 1996. Crystal structure of the Aequorea victoria green fluorescent protein, Science, 273, 1392-1395. 
Pakhomov, A. A., Martynova, N. Y., Gurskaya, N. G., Balashova, T. A., and Martynov, V. I. 2004. Photoconversion of the chromophore of a fluorescent protein from Dendronephthya sp., Biochemistry (Moscow), 69, 901-908.

Patterson, G. H. 2004. A new harvest of fluorescent proteins, Nature Biotechnology, 22, 1524-1525.

Patterson, G. H., Day, R. N., and Piston, D. W. 2001. Fluorescent protein spectra, Journal of Cell Science, 114, 837-838.

Patterson, G. H., Knobel, S. M., Sharif, W. D., Kain, S. R., and Piston, D. W. 1997. Use of the green fluorescent protein and its mutants in quantitative fluorescence microscopy, Biophysical Journal, 73, 2782-2790.

Patterson, G. H. and Lippincott-Schwartz, J. 2002a. A photoactivatable GFP for selective photolabeling of proteins and cells, Science, 297, 1873-1877.

Patterson, G. H. and Lippincott-Schwartz, J. 2002b. Selective photolabeling of proteins using photoactivatable GFP, Methods, 32, 445-450.

Patterson, G. H., Piston, D. W., and Barisas, B. G. 2000. Förster distances between green fluorescent protein pairs, Analytical Biochemistry, 284, 438-440.

Periasamy, A. and Day, R. N. 1998. FRET imaging of Pit-1 protein interactions in living cells, Journal of Biomedical Optics, 3, 154-160.

Peterson, J., Wilmann, P. G., Beddoe, T., Oakley, A. J., Devenish, R. J., Prescott, M., and Rossjohn, J. 2003. The 2.0- $\AA$ crystal structure of eqFP611, a far red fluorescent protein from the sea anemone Entacmaea quadricolor, Journal of Biological Chemistry, 278, 44626-44631.

Phillips, G. N. 2006. The three-dimensional structure of green fluorescent protein and its implications for function and design. In Green Fluorescent Protein: Properties, Applications, and Protocols, $2^{\text {nd }}$ ed. (M. Chalfie and S. R. Kain, eds.) pp. 67-82. Wiley-Interscience, New York.

Potter, S. M. 1996. Vital imaging: Two photons are better than one, Current Biology, 6, 1595-1598.

Prasher, D. C., Eckenrode, V. K., Ward, W. W., Prendergast, F. G., and Cormier, M. J. 1992. Primary structure of the Aequorea victoria green-fluorescent protein, Gene, 111, 229-233.

Rajfur, Z., Roy, P., Otey, C., Romer, L., and Jacobson, K. 2002. Dissecting the link between stress fibers and focal adhesions by CALI with EGFP fusion proteins, Nature Cell Biology, 4, 286-293.

Rehm, M., Duessmann, H., Jaenicke, R. U., Tavare, J. M., Koegel, D., and Prehn, J. H. M. 2002. Singlecell fluorescence resonance energy transfer analysis demonstrates that caspase activation during apoptosis is a rapid process, Journal of Biological Chemistry, 277, 24506-24514.

Remington, S. J., Wachter, R. M., Yarbrough, D. K., Branchaud, B., Anderson, D. C., Kallio, K., and Lukyanov, K. A. 2005. zFP538, a yellow-fluorescent protein from Zoanthus, contains a novel threering chromophore, Biochemistry, 44, 202-212.

Richards, B., Zharkikh, L., Hsu, F., Dunn, C., Kamb, A., and Teng, D. H. 2002. Stable expression of Anthozoa fluorescent proteins in mammalian cells, Cytometry, 48, 106-112.

Rizzo, M. A., Magnuson, M. A., Drain, P. F., and Piston, D. W. 2002. A functional link between glucokinase binding to insulin granules and conformational alterations in response to glucose and insulin, Journal of Biological Chemistry, 277, 34168-34175.

Rizzo, M. A. and Piston, D. W. 2005a. Fluorescent protein tracking and detection. In Live Cell Imaging: A Laboratory Manual (R. D. Goldman and D. L. Spector, eds.) pp. 3-23. Cold Spring Harbor Laboratory Press, Cold Spring Harbor, New York.

Rizzo, M. A. and Piston, D. W. 2005b. High-contrast imaging of fluorescent protein FRET by fluorescence polarization microscopy, Biophysical Journal, 88, L14-L16.

Rizzo, M. A., Springer, G. H., Granada, B., and Piston, D. W. 2004. An improved cyan fluorescent protein variant useful for FRET, Nature Biotechnology, 22, 445-449.

Rizzuto, R., Brini, M., De Giorgi, F., Rossi, R., Hein, R., Tsien, R. Y., and Pozzan, T. 1996. Double labeling of subcellular structures with organelle-targeted GFP mutants in vivo, Current Biology, 6, 183-188.

Romoser, V. A., Hinkle, P. M., and Persechini, A. 1997. Detection in living cells of calcium-dependent changes in the fluorescence emission of an indicator composed of two green fluorescent protein variants linked by a calmodulin-binding sequence, Journal of Biological Chemistry, 272, 1327013274.

Salih, A., Larkum, A., Cox, G., Kuhl, M., and Hoegh-Buldberg, O. 2000. Fluorescent pigments in corals are photoprotective, Nature, 408, 850-853. 
Schenk, A., Ivanchenko, S., Roecker, C., Wiedenmann, J., and Nienhaus, G. U. 2004. Photodynamics of red fluorescent proteins studied by fluorescence correlation spectroscopy, Biophysical Journal, 86, 384-394.

Shagin, D. A., Barsova, E. V., Yanushevich, Y. G., Fradkov, A. F., Lukyanov, K. A., Labas, Y. A., Semenova, T. N., Ugalde, J. A., Meyers, A., Nunez, J. M., Widder, E. A., Lukyanov, S. A., and Matz, M. V. 2004. GFP-like proteins as ubiquitous metazoan superfamily: Evolution of functional features and structural complexity, Molecular Biology and Evolution, 21, 841-850.

Shaner, N. C., Campbell, R. E., Steinbach, P. A., Giepmans, B. N. G., Palmer, A. E., and Tsien, R. Y. 2004. Improved monomeric red, orange and yellow fluorescent proteins derived from Discosoma sp. red fluorescent protein, Nature Biotechnology, 22, 1567-1572.

Shaner, N. C., Steinbach, P. A., and Tsien, R. Y. 2005. A guide to choosing fluorescent proteins, Nature Methods, 2, 905-909.

Sheff, M. A. and Thorn, K. S. 2004. Optimized cassettes for fluorescent protein tagging in Saccharomyces cerevisiae, Yeast, 21, 661-670.

Sheridan, D. L. and Hughes, T. E. 2004. A faster way to make GFP-based biosensors: Two new transposons for creating multicolored libraries of fluorescent fusion proteins, BMC Biotechnology, 4, $1-17$.

Shimomura, O., Johnson, F. H., and Saiga, Y. 1962. Extraction, purification, and properties of aequorin, a bioluminescent protein from the luminous hydromedusan, Aequorea, Journal of Cellular and Comparative Physiology, 59, 223-239.

Shkrob, M. A., Yanushevich, Y. G., Chudakov, D. M., Gurskaya, N. G., Labas, Y. A., Poponov, S. Y., Mudrik, N. N., Lukyanov, S., and Lukyanov, K. A. 2005. Far-red fluorescent proteins evolved from a blue chromoprotein from Actinia equine, Biochemical Journal, 392, 649-654.

Shyu, Y. J., Liu, H., Deng, X., and Hu, C. D. 2006. Identification of new fluorescent protein fragments for bimolecular fluorescence complementation analysis under physiological conditions, BioTechniques, 40, 61-66.

Silva, E., Salim-Hanna, M., Edwards, A. M., Becker, M. I., and De Ioannes, A.E. 1991. A light-induced tryptophan-riboflavin binding: Biological implications, Advances in Experimental Medicine and Biology, 289, 33-48.

Spierenburg, G. T., Oerlemans, F. T., van Laarhoven, J. P., and de Bruyn, C. H. 1984. Phototoxicity of N2-hydroxyethylpiperazine-N'-2-ethanesulfonic acid-buffered culture media for human leukemic cell lines, Cancer Research, 44, 2253-2254.

Stephens, D. J. and Allan, V. J. 2003. Light microscopy techniques for live cell imaging, Science, 300, 8286.

Sturman, D. A., Shakiryanova, D., Hewes, R., Deitcher, D., and Levitan, E. S. 2006. Nearly neutral secretory vesicles in Drosophila nerve terminals, Biophysical Journal, 90, in press.

Tavaré, J. M., Fletcher, L. M., and Welsh, G. I. 2001. Using green fluorescent protein to study intracellular signaling, Journal of Endocrinology, 170, 297-306.

Ting, A. Y., Kain, K. H., Klemke, R. L., and Tsien, R. Y. 2001. Genetically encoded fluorescent reporters of protein tyrosine kinase activities in living cells, Proceedings of the National Academy of Sciences, USA, 98, 15003-15008.

Tinnefeld, P. and Sauer, M. 2005. Branching out of single-molecule fluorescence spectroscopy: Challenges for chemistry and influence on biology, Angewandte Chemie, 44, 2642-2671.

Tour, O., Meijer, R. M., Zacharias, D. A., Adams, S. R., and Tsien, R. Y. 2003. Genetically targeted chromophore-assisted light inactivation, Nature Biotechnology, 21, 1505-1508.

Tsien, R. Y. 1998. The green fluorescent protein, Annual Review of Biochemistry, 67, 509-544.

Tsien, R. Y. 2005. Building and breeding molecules to spy on cells and tumors, Federation of the European Biochemical Societies (FEBS) Letters, 579, 927-932.

Tsuboi, T. and Rutter, G. A. 2003. Insulin secretion by "kiss-and-run" exocytosis in clonal pancreatic islet beta-cells, Biochemical Society Transactions, 31, 833-836.

Tsutsui, H., Karasawa, S., Shimizu, H., Nukina, N., and Miyawaki, A. 2005. Semi-rational engineering of a coral fluorescent protein into an efficient highlighter, European Molecular Biology Organization (EMBO) Reports, 6, 233-238.

Verkhusha, V. V. and Lukyanov, K. A. 2004. The molecular properties and applications of Anthozoa fluorescent proteins and chromoproteins, Nature Biotechnology, 22, 289-296. 
Verkhusha, V. V. and Sorkin, A. 2005. Conversion of the monomeric red fluorescent protein into a photoactivatable probe, Chemistry and Biology, 12, 279-285.

Vrzheshch, P. V., Akovbian, N. A., Varfolomeyev, S. D., and Verkhusha, V. V. 2000. Denaturation and partial renaturation of a tightly tetramerized DsRed protein under mildly acidic conditions, Federation of the European Biochemical Societies (FEBS) Letters, 487, 203-208.

Wachter, R. M., Elsliger, M. A., Kallio, K., Hanson, G. T., and Remington, S. J. 1998. Structural basis of spectral shifts in the yellow-emission variants of green fluorescent protein, Structure, 6, 1267-1277.

Wallrabe, H. and Periasamy, A. 2005. Imaging protein molecules using FRET and FLIM microscopy, Current Opinion in Biotechnology, 16, 19-27.

Wang, L., Jackson, W. C., Steinbach, P. A., and Tsien, R. Y. 2004. Evolution of new nonantibody proteins via iterative somatic hypermutation, Proceedings of the National Academy of Sciences, USA, 101, 16745-16749.

Ward, W. W. 2006. Biochemical and physical properties of green fluorescent protein. In Green Fluorescent Protein: Properties, Applications, and Protocols, $2^{\text {nd }}$ ed. (M. Chalfie and S. R. Kain, eds.) pp. 39-65. Wiley-Interscience, New York.

Ward, W. W. and Cormier, M. J. 1979. An energy transfer protein in coelenterate bioluminescence: Characterization of the Renilla green-fluorescent protein (GFP), Journal of Biological Chemistry, 254, 781-788.

Wiedenmann, J., Ivanchenko, S., Oswald, F., Schmitt, F., Rocker, C., Salih, A., Spindler, K., and Nienhaus, G. U. 2004. EosFP, a fluorescent marker protein with UV-inducible green-to-red fluorescence conversion, Proceedings of the National Academy of Sciences, USA, 101, 15905-15910.

Wiedenmann, J., Schenk, A., Roecker, C., Girod, A., Spindler, K. D., and Nienhaus, G. U. 2002. A far-red fluorescent protein with fast maturation and reduced oligomerization tendency from Entacmaea quadricolor (Anthozoa, Actinaria), Proceedings of the National Academy of Sciences, USA, 99, 1164611651.

Wiedenmann, J., Vallone, B., Renzi, F., Nienhaus, K., Ivanchenko, S., Roecker, C., and Nienhaus, G. U. 2005. Red fluorescent protein eqFP611 and its genetically engineered dimeric variants, Journal of Biomedical Optics, 10, 1-7.

Wiehler, J., von Hummel, J., and Steipe, B. 2001. Mutants of Discosoma red fluorescent protein with a GFP-like chromophore, Federation of the European Biochemical Societies (FEBS) Letters, 487, 384389.

Yang, T. T., Sinai, P., Green, G., Kitts, P. A., Chen, Y. T., Lybarger, L., Cervenak, R., Patterson, G. H., Piston, D. W., and Kain, S. R. 1998. Improved fluorescence and dual color detection with enhanced blue and green variants of the green fluorescent protein, Journal of Biological Chemistry, 273, 82128216.

Yanushevich, Y. G., Staroverov, D. B., Savitsky, A. P., Fradkov, A. F., Gurskaya, N. G., Bulina, M. E., Lukyanov, K. A., and Lukyanov, S. A. 2001. A strategy for the generation of non-aggregating mutants of Anthozoa fluorescent proteins, Federation of the European Biochemical Societies (FEBS) Letters, 511, 11-14.

Yarbrough, D., Wachter, R. M., Kallio, K., Matz, M. V., and Remington, S. J. 2001. Refined crystal structure of DsRed, a red fluorescent protein from coral, at $2.0 \AA$ resolution, Proceedings of the National Academy of Sciences, USA, 98, 462-467.

Zaccolo, M. 2004. Use of chimeric fluorescent proteins and fluorescence resonance energy transfer to monitor cellular responses, Circulation Research, 94, 866-873.

Zacharias, D. A. 2002. Sticky caveats in an otherwise glowing report: Oligomerizing fluorescent proteins and their use in cell biology, Science STKE, 131, pe23, 1-3.

Zacharias, D. A. and Tsien, R. Y. 2006. Molecular biology and mutation of green fluorescent protein. In Green Fluorescent Protein: Properties, Applications, and Protocols, $2^{\text {nd }}$ ed. (M. Chalfie and S. R. Kain, eds.) pp. 83-120. Wiley-Interscience, New York.

Zacharias, D. A., Violin, J. D., Newton, A. C., and Tsien, R. Y. 2002. Partitioning of lipid-modified monomeric GFPs into membrane microdomains of live cells, Science, 296, 913-916.

Zagranichny, V. E., Rudenko, N. V., Gorokhovatsky, A. Y., Zakharov, M. V., Shenkarev, Z. O., Balashova, T. A., and Arseniev, A. S. 2004. zFP538, a yellow fluorescent protein from coral, belongs to the DsRed subfamily of GFP-like proteins but possesses the unexpected site of fragmentation, Biochemistry, 43, 4764-4772. 
Zapata-Hommer, O. and Griesbeck, O. 2003. Efficiently folding and circular permuted variants of the Sapphire mutant of GFP, BMC Biotechnology, 3, 5-11.

Zhang, J., Campbell, R. E., Ting, A. Y., and Tsien, R. Y. 2002. Creating new fluorescent probes for cell biology, Nature Reviews Molecular Cell Biology, 3, 906-918.

Zhang, J., Ma, Y., Taylor, S. S., and Tsien, R. Y. 2001. Genetically encoded reporters of protein kinase A activity reveal impact of substrate tethering, Proceedings of the National Academy of Sciences, USA, 98, 14997-15002.

Zimmer, M. 2002. Green fluorescent protein (GFP): Applications, structure, and related photophysical behavior, Chemical Reviews, 102, 759-781.

Zumbusch, A. and Jung, G. 2000. Single molecule spectroscopy of the green fluorescent protein: A critical assessment, Single Molecules, 1, 261-270. 